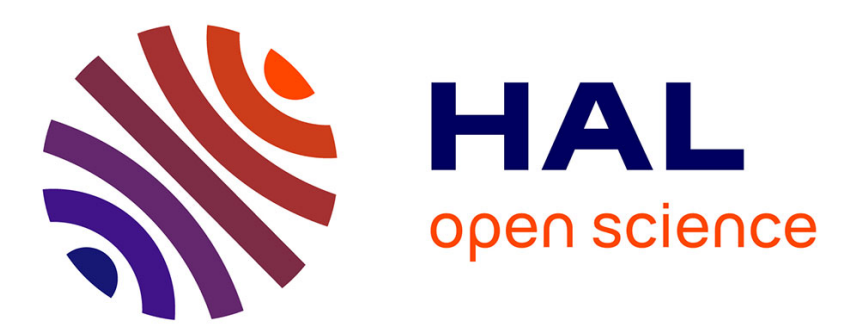

\title{
A Unifying Approach for Disturbance Cancellation and Target Detection in Passive Radar Using OFDM
}

\author{
Gilles Chabriel, Ghislain Gassier, Jean Barrère, Francoise Briolle, Claude
}

Jauffret

\section{To cite this version:}

Gilles Chabriel, Ghislain Gassier, Jean Barrère, Francoise Briolle, Claude Jauffret. A Unifying Approach for Disturbance Cancellation and Target Detection in Passive Radar Using OFDM. IEEE Transactions on Signal Processing, 2016, 64 (22), pp.5959 - 5971. 10.1109/TSP.2016.2600511 . hal01793471

\section{HAL Id: hal-01793471 \\ https://hal.science/hal-01793471}

Submitted on 16 May 2018

HAL is a multi-disciplinary open access archive for the deposit and dissemination of scientific research documents, whether they are published or not. The documents may come from teaching and research institutions in France or abroad, or from public or private research centers.
L'archive ouverte pluridisciplinaire HAL, est destinée au dépôt et à la diffusion de documents scientifiques de niveau recherche, publiés ou non, émanant des établissements d'enseignement et de recherche français ou étrangers, des laboratoires publics ou privés. 


\title{
A Unifying Approach for Disturbance Cancellation and Target Detection in Passive Radar using OFDM
}

\author{
G. Gassier, G. Chabriel, J. Barrère, F. Briolle and C. Jauffret
}

\begin{abstract}
This paper addresses the problem of mobile target detection in multipath scenarios with a passive radar using DVB-T transmitters of opportunity. For such emissions, it has been shown the interest in implementing "mismatched" correlators, reducing both the zero Doppler contribution (ZDC) masking effects and the false alarm rate. A very efficient mismatched reference signal is obtained with the reciprocal filter (or inverse filter) which consist in a modulus frequential equalization of the transmitted signal.

We propose here to revisit the reciprocal filter based correlator and to reinterpret it as a so-called Doppler channel detector (CHAD). This new interpretation allows a direct rejection of the ZDC, unifying in one and the same step the main disturbance mitigation and the detector construction. We provide a statistical theoretical study of the performance and a comparison with the matched correlator $i . e$. the classical cross-ambiguity function (CAF). We demonstrate that CHAD has a random pedestal (a clutter floor level) significantly lower than that of the classical CAF for low Doppler frequency shifts. Numerical experiments on simulated and real data as well validate the mathematical derivations.
\end{abstract}

Index Terms-Passive bistatic radar (PBR), passive covert radar (PCR), passive coherent location (PCL), digital video broadcasting-terrestrial (DVB-T), orthogonal frequency division multiplexing (OFDM), crossambiguity function (CAF), mismatched filter, reciprocal filter, clutter.

\section{INTRODUCTION}

A Passive bistatic radar (PBR) exploits illuminators of opportunity to detect moving targets from their Doppler-shifted frequency echoes (see e.g. [1], [2], [3]). We focus here on the very widespread digital video broadcasting - terrestrial (DVB-T) transmitters. These emissions are interesting from a signal processing point of view: the bandwidth (7.6 MHz in Europe) allows the target position to be theoretically estimated with a good resolution (20 $\mathrm{m}$ at best); the underlying orthogonal frequency division multiplexing (OFDM) allows the broadcast signal detection through a multipath channel

G. Chabriel, J. Barrère and C. Jauffret are with Aix Marseille Université, CNRS, Université de Toulon, IM2NP UMR 7334, CS 60584 - 83041 Toulon Cedex 9, France (e-mail: gilles.chabriel@im2np.fr; jean.barrere@im2np.fr; claude.jauffret@im2np.fr).

G. Gassier is with Aix Marseille Université, CNRS, Université de Toulon, IM2NP UMR 7334, CS 60584 - 83041 Toulon cedex 9, France and with Ecole de l'Air \& CReA, B.A. 701, 13661 Salon-Air, France (ghislain.gassier@gmail.com).

F. Briolle is with Aix Marseille Université, CNRS, Université de Toulon, CPT UMR 7332, Campus de Luminy, Case 907, 13288 Marseille cedex 9, France and with Ecole de l'Air \& CReA, B.A. 701, 13661 Salon-Air, France (francoise.briolle@gmail.com).
[4], and an antenna dedicated to the reference signal can then be avoided [5].

A main difficulty concerns the presence of the very powerful line-of-sight (LOS) path and its rooted replicas. In the surveillance signal, this strong continuous zero Doppler contribution (ZDC) may mask the echoes of the smallest radar cross-section (RCS) targets in the range-frequency plane of the cross-ambiguity function $(\mathrm{CAF})$. More precisely, since the integration time is necessarily limited ${ }^{1}$, the dominant paths induce both secondary lobes (essentially along the Doppler axis) and a random pedestal abusively called clutter floor, whose magnitude can be higher than the peaks of interest.

Since, for moving targets, the observation time must remain reasonably low, a $\mathrm{ZDC}$ rejection preprocessing is often performed [6], [7], [8], [9].

Classically, a weighted set of time-delayed versions of the broadcast signal is fitted, in the least-squares (LS) sense, to the surveillance signal [6], [7] then subtracted. The main drawbacks in the aforementioned LS-based ZDC rejection are a quite heavy computational and memory loads. To decrease the memory load, a batch implementation is also proposed in [6]: the $\mathrm{ZDC}$ is estimated using consecutive smaller signal portions instead of the whole signal duration. Unfortunately, in this case, remaining correlations with low Doppler sources conduct to a detection loss on both sides near the null Doppler axis. Moreover, for DVB-T signals, the periodical insertion of boosted pilot symbols, used for synchronization and channel equalization, gives rise to multiple ghost-peaks "spamming" the CAF output [10], [11].

An alternative method using the OFDM structure and reducing both the memory load and the computational complexity, was proposed by D. Poullin in [8]. The surveillance signal is projected orthogonally to the frequency subspace corresponding to the ZDC. The projector is cleverly approximated by a set of inverse covariance matrices evaluated for each OFDM subcarrier. Let us also mention [9] where a low complexity ZDC rejection method based on the $\mathrm{CP}-\mathrm{OFDM}$ structure of the surveillance signal is described.

Note that in all the previous approaches, despite the ZDC mitigation, a stronger target may mask a weaker one as well. Such dominant echoes must then specifically

\footnotetext{
${ }^{1}$ One has to respect $T \ll \frac{c}{B v}$ where $T, B, v$ and $c$ are respectively the integration time, the signal bandwidth, the target bistatic speed and the celerity of light.
} 
be removed by a multistage elimination process as it is proposed in [6].

Beside these ZDC mitigation studies, some authors suggested to perform a CAF using so-called "mismatched" versions of the illumination signal as a new reference: first, Glende [12] proposed, for systems working with analog $\mathrm{TV}$, to use a modulus equalized (a reciprocal filtered) version of the transmitted signal. For DVB-T emissions, Palmer et al. [10] use some mismatched reference signal to reduce the false alarms due to pilot data. Then, they extended their approach [13] by using the reciprocal filter associated to a circular correlator. Using a particular sampling along the Doppler dimension (note that such a sampling was already proposed by Berger et al. [14] for CAF on DAB signals), they obtain a well-performing detector without the need of a ZDC mitigation pre-stage. We will denote this method by the inverse filter approach (IFA).

As main contributions, we propose in this paper, a new interpretation of IFA called "channel detector" (CHAD) and a theoretical study of its performance. The principle of this new approach is to estimate the so-called Doppler channel due to paths with a same Doppler shift; the corresponding impulse response is directly used to detect the underlying echoes. This detector is shown to be theoretically robust to clutter and we prove that its random pedestal is generally lower than that of the classical CAF.

The paper is organized as follows: Section II presents the notion of time-varying Doppler channel associated with the frequency $\nu$ characterized by its frequency response $H_{\nu}$ or equivalently by the impulse response $h_{\nu}$.

CHAD approach is presented in Section III: an estimation of $\left|h_{\nu}\right|^{2}$ is used to detect the set of echoes with the same Doppler-shifted frequency $\nu$. Theoretical performance comparisons between CHAD and CAF are presented.

The validity of the mathematical derivations is illustrated by numerical experiments on simulated and experimental data (Section VI and Section VII).

\section{Notion of Doppler Channel}

A typical passive bistatic radar scenario is depicted in Fig. 1, where a transmitter illuminates a scene composed of different static scatterers due to topography, buildings ..., and one or several moving reflectors of interest, namely the targets. A wide spatial-aperture receive antenna collects the radiations from the different paths.

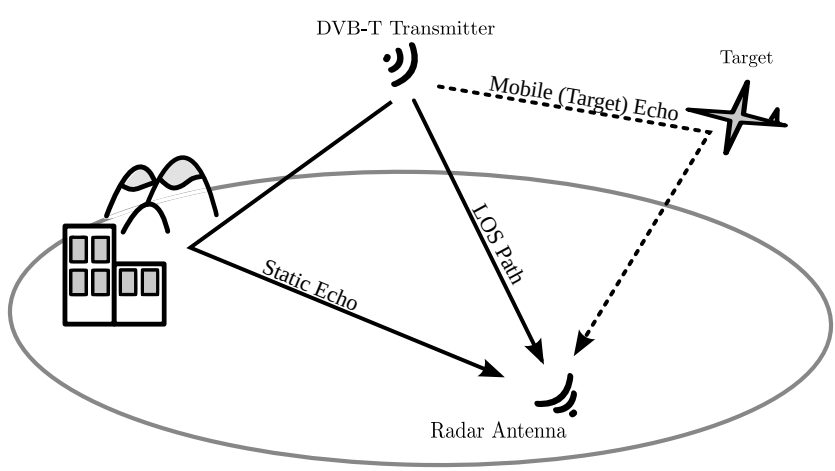

Fig. 1. Passive bistatic radar typical scene

Let us consider a passband high frequency broadcast signal of opportunity and let us denote by $s_{e}$ its baseband version. The observation time is here assumed to be sufficiently small so that the Doppler dilatation/compression effect can be neglected. Due to the multipath propagation channel, the baseband received signal $y(t)$ then corresponds to a noisy linear combination of time-delayed replicas of $s_{e}(t)$, with frequency shifts depending on reflector velocities.

Denoting by $\mathcal{I}_{I}$, the index set $\{0,1, \ldots, I-1\}$, the received signal can then be classically modeled as:

$$
y(t)=\sum_{i \in \mathcal{I}_{I}} \alpha_{i} s_{e}\left(t-\tau_{i}-t_{0}\right) \mathrm{e}^{\jmath 2 \pi f_{\mathrm{p}_{i}} t}+\eta(t),
$$

where:

- $i$ is the reflector/path index varying from 1 to $I-1$; $i=0$ being reserved for the LOS path,

- $t_{0}$ is the LOS path propagation delay (from the transmitter to the radar antenna),

- $\tau_{i}$ is the relative propagation delay such that $\tau_{i}+t_{0}$ corresponds to the propagation delay of the path $i$ (i.e. from the transmitter to the receive antenna, via the $i^{\text {th }}$ reflector),

- $f_{\mathrm{p}_{i}}$ is the Doppler-shifted frequency corresponding to the path $i$,

- $\alpha_{i}$ are factors depending on positions, RCS of the different reflectors, ...

- $\eta(t)$ corresponds to the additive white Gaussian noise (AWGN) channel.

The LOS path being indexed by 0 , one has necessarily $\tau_{0}=0$ and $f_{\mathrm{p}_{0}}=0$.

Unlike conventional active radar systems, the emitted signal $s_{e}$ is here not directly available. Nevertheless, with a DVB-T emission, one can perfectly recover the transmitted data and consequently synthesize a baseband temporal version of the LOS received signal. This synthetic signal, referred as the "reference signal", is denoted by $s$ in the sequel. The surveillance signal can now be expressed as a 
function of $s$ :

$$
y(t)=\sum_{i \in \mathcal{I}_{I}} \alpha_{i} s\left(t-\tau_{i}\right) \mathrm{e}^{\jmath 2 \pi f_{\mathrm{p}_{i}} t}+\eta(t) .
$$

Assuming that $F$ different Doppler-shifted frequencies are observed, we gather the paths with the same Dopplershifted frequency, say $f_{j}$, and define the corresponding index sets

$$
\mathcal{J}_{f_{j}}=\left\{i \in \mathcal{I}_{I} \mid f_{\mathrm{p}_{i}}=f_{j}\right\}, j \in \mathcal{I}_{F}
$$

It can then be considered that the reference signal is received through $F$ distinct linear time-varying channels, called subsequently Doppler channels and the observation (2) is rewritten as

$$
y(t)=\sum_{j \in \mathcal{I}_{F}}\left(h_{f_{j}} * s\right)(t) \mathrm{e}^{\jmath 2 \pi f_{j} t}+\eta(t)
$$

where the impulse response $h_{f_{j}}(t)$ :

$$
h_{f_{j}}(t) \triangleq \sum_{i \in \mathcal{J}_{f_{j}}} \alpha_{i} \delta\left(t-\tau_{i}\right)
$$

characterizes the time-invariant part of the $j^{\text {th }}$ Doppler channel. The particular channel $h_{0}$ (corresponding to the null Doppler frequency $f_{0}=0$ ), will be called the static channel.

\section{CHAD (DoppleR CHANNEL Detection)}

\section{A. Recall of DVB-T Signal Main Features}

The DVB-T analog signal is a sequence of so-called CPOFDM symbols, of duration $T_{s}$. Each symbol is composed of two parts: the useful part of duration $T_{u}$ and a redundant part of duration $T_{c p}$, called the cyclic prefix $(\mathrm{CP})$. The useful part of a symbol $m$ is composed of $K$ orthogonal subcarriers $f_{k}=\frac{k}{T_{u}}$ carrying a complex data $d_{k}^{m}$.

Most of the subcarriers are modulated by information data $d_{k}^{m}$ which can only take $Q$ complex discrete values depending on the $Q$-ary QAM modulation used. Some of them are modulated by a sequence of known data: the pilot data. Finally, a set of low and high subcarrier frequencies are not modulated ensuring that the overall bandwidth $B$ stays within $K / T_{u}$. We denote by $\mathcal{E}_{K}=\left\{K_{\mathrm{m}}, \ldots, K_{\mathrm{M}}\right\} \subset \mathcal{I}_{K}$, the index subset of the effectively modulated subcarriers ${ }^{2}$. The cardinality of $\mathcal{E}_{K}$ is denoted $\# \mathcal{E}_{K}$.

The cyclic prefix is obtained by copying the last part of the symbol and attaching it in front of the useful part. Its duration $T_{c p}$ is chosen to be greater than the echo delays, to avoid intersymbol (ISI) and intercarrier (ICI) interferences [4].

The temporal expression of any baseband emitted CPOFDM symbol $m$ is then:

$$
s^{m}\left(t^{\prime}\right)=\frac{1}{K} \sum_{k \in \mathcal{E}_{K}} d_{k}^{m} \mathrm{e}^{\jmath 2 \pi \frac{k}{T_{u}} t^{\prime}},
$$

\footnotetext{
${ }^{2}$ Note that in the DVB-T standard, $\# \mathcal{E}_{K} / K \approx 0.83$.
}

where $t^{\prime}$ is a local time variable such as $-T_{c p} \leq t^{\prime}<T_{u}$. During the observation time, the entire baseband emitted signal forms a sequence of $M$ symbols we will denote by

$$
s(t)=\left(s^{0}\left(t^{\prime}\right), \ldots, s^{M-1}\left(t^{\prime}\right)\right)=\left(s^{m}\left(t^{\prime}\right)\right)_{m \in \mathcal{I}_{M}}
$$

where $t$ is now the global time variable $0 \leq t<M T_{s}$.

Assuming an error-free DVB-T demodulation, the data $d_{k}^{m}, m \in \mathcal{I}_{M}, k \in \mathcal{E}_{K}$, are assumed known throughout the remainder of the paper.

\section{B. Detection Principle}

Since each $f_{j}$-Dopplerized target is revealed by a peak (ideally a Dirac pulse, see (4)) in the impulse response $h_{f_{j}}$ corresponding to its Doppler channel, the main idea is to estimate such an impulse response from the sampled received signal $y[k]$ and to use it as a detection function. Introducing the under test Doppler frequency $\nu$, the estimation of $h_{\nu}$ needs the underlying Doppler channel to be stationarized. We then define the corresponding Doppler compensated observation:

$$
y_{\nu}(t) \triangleq y(t) \mathrm{e}^{-\jmath 2 \pi \nu t} .
$$

Using (3), $y_{\nu}(t)$ becomes

$$
y_{\nu}(t)=\sum_{j \in \mathcal{I}_{F}}\left(h_{f_{j}} * s\right)(t) \mathrm{e}^{\jmath 2 \pi\left(f_{j}-\nu\right) t}+\eta_{\nu}(t),
$$

where $\eta_{\nu}(t)$ is the frequency-shifted noise contribution.

Introducing the index set $\mathcal{J}_{\nu}=\left\{i \in \mathcal{I}_{I} / f_{\mathrm{p}_{i}}=\nu\right\}$, let us now rewrite (8) as

$$
y_{\nu}(t)=\bar{y}_{\nu}(t)+\tilde{y}_{\nu}(t)+\eta_{\nu}(t),
$$

where the so-called static part $\bar{y}_{\nu}(t)$ of $y_{\nu}(t)$, if it exists $(i . e$. if $\mathcal{J}_{\nu} \neq \emptyset$ ), gathers the contributions of all paths having a null Doppler shift after compensation (the set of paths with $f_{\mathrm{p}_{i}}=\nu$ before compensation), while the dynamic part $\tilde{y}_{\nu}$ collects the remaining paths. One has

$$
\begin{aligned}
\bar{y}_{\nu}(t) & =\left(h_{\nu} * s\right)(t) \\
\text { where (ideally) } h_{\nu}(t) & =\sum_{i \in \mathcal{J}_{\nu}} \alpha_{i} \delta\left(t-\tau_{i}\right) .
\end{aligned}
$$

Let us assume that $t=0$ corresponds to the beginning of the useful part of an OFDM symbol coming up the direct path $^{3}$. The compensated observed signal can then be cut out into blocks of duration $T_{s}$ :

$$
y_{\nu}(t)=\left(y_{\nu}^{m}\left(t^{\prime}\right)\right)_{m \in \mathcal{I}_{M}}, \quad 0 \leq t^{\prime}<T_{s} .
$$

Thanks to the CP-OFDM structure of the signal and assuming that the delays $\tau_{i}, i \in \mathcal{J}_{\nu}$ are less than the $\mathrm{CP}$ duration $^{4}$, it can be shown from (4), (5) and (10) that the static part of each block yields

\footnotetext{
${ }^{3} \mathrm{CP} /$ pilot-aided standard synchronization.

${ }^{4}$ This assumption may seem restrictive but it corresponds anyway to bistatic distances up to $70 \mathrm{~km}\left(8 \mathrm{~K}\right.$ mode, $\left.T_{c p}=T_{u} / 4\right)$. Moreover,
} we will see that this assumption can be reasonably overridden. 


$$
\bar{y}_{\nu}^{m}\left(t^{\prime}\right)=\sum_{k \in \mathcal{E}_{K}} H_{\nu}[k] d_{k}^{m} \mathrm{e}^{\jmath 2 \pi \frac{k}{T_{u}} t^{\prime}}, \quad 0 \leq t^{\prime}<T_{u},
$$

where the $\# \mathcal{E}_{K}$ complex coefficients $H_{\nu}[k]$ correspond to the frequency response of the $\nu$-Doppler channel at frequencies $\frac{k}{T_{u}}$ :

$$
H_{\nu}[k]=\sum_{i \in \mathcal{J}_{\nu}} \alpha_{i} \exp \left(-\jmath 2 \pi \frac{k}{T_{u}} \tau_{i}\right), \quad k \in \mathcal{E}_{K} .
$$

Let us sample the compensated received signal at the data symbol period $T_{u} / K$. Using $t^{\prime}=n^{\prime} T_{u} / K$, we can write for each block $y_{\nu}^{m}\left[n^{\prime}\right], m \in \mathcal{I}_{M}{ }^{5}$ :

$$
\begin{array}{r}
y_{\nu}^{m}\left[n^{\prime}\right]=\sum_{k \in \mathcal{E}_{K}} H_{\nu}[k] d_{k}^{m} \mathrm{e}^{\jmath 2 \pi \frac{k n^{\prime}}{K}}+\tilde{y}_{\nu}^{m}\left[n^{\prime}\right]+\eta_{\nu}^{m}\left[n^{\prime}\right], \\
n^{\prime} \in \mathcal{I}_{K} .
\end{array}
$$

Applying a $K$-points discrete Fourier transform (DFT) on the previous compensated received block, one obtains the following spectral representation:

$$
Y_{\nu}^{m}[k]=H_{\nu}[k] d_{k}^{m}+\tilde{Y}_{\nu}^{m}[k]+\Xi_{\nu}^{m}[k], \quad k \in \mathcal{E}_{K},
$$

with $\tilde{Y}_{\nu}^{m} \triangleq \operatorname{DFT}\left\{\tilde{y}_{\nu}^{m}\right\}$ and $\Xi_{\nu}^{m} \triangleq \operatorname{DFT}\left\{\eta_{\nu}^{m}\right\}$.

It is proved (Subsection A of the Appendix) that $\forall k \in$ $\mathcal{E}_{K}$ and $\forall \nu$,

$$
\begin{aligned}
& \lim _{M \rightarrow+\infty} \frac{1}{M} \sum_{m \in \mathcal{I}_{M}} \frac{\tilde{Y}_{\nu}^{m}[k]}{d_{k}^{m}}=0 \\
& \text { and } \lim _{M \rightarrow+\infty} \frac{1}{M} \sum_{m \in \mathcal{I}_{M}} \frac{\Xi_{\nu}^{m}[k]}{d_{k}^{m}}=0 .
\end{aligned}
$$

Hence,

$$
\hat{H}_{\nu}[k] \triangleq \frac{1}{M} \sum_{m \in \mathcal{I}_{M}} \frac{Y_{\nu}^{m}[k]}{d_{k}^{m}}, \quad k \in \mathcal{E}_{K}
$$

is an asymptotically unbiased estimator of the $\nu$-Doppler channel frequency response $H_{\nu}$. Thereafter, an estimation of the corresponding impulse response $\hat{h}_{\nu}$ is obtained by a simple Inverse Discrete Fourier transform (IDFT)

$$
\hat{h}_{\nu}[n]=\frac{1}{K} \sum_{n \in \mathcal{I}_{K}} \hat{H}_{\nu}[k] \mathrm{e}^{j 2 \pi \frac{k n}{K}} .
$$

Finally, a parametric sweep over the Doppler frequency shift $\nu$, allows to define the so-called channel detector $C H A D$ through the following range-frequency function:

$$
\mathcal{D}(\tau, \nu) \triangleq\left|\hat{h}_{\nu}[n]\right|^{2}, \text { with } \tau=n T_{u} / K .
$$

Note that, since a DVB-T signal is bandlimited, the coefficients $\hat{H}_{\nu}[k], \quad k \in \mathcal{I}_{K} \backslash \mathcal{E}_{K}$ are non-observable and have then to be replaced by zero. This leads to (squared) sinc-shaped detection peaks (instead of Dirac pulses), limiting the resolution along the range axis.

\footnotetext{
${ }^{5}$ In practice, $y_{\nu}^{m}\left[n^{\prime}\right]$ is built from the sampled version of the received signal, that is to say $y_{\nu}^{m}\left[n^{\prime}\right]=y^{m}\left[n^{\prime}\right] \mathrm{e}^{-\jmath 2 \pi \nu\left(n^{\prime}+m\right) \frac{T_{u}}{K}}$.
}

Finally, for the Doppler-shifted frequencies $\nu$ we are interested in ${ }^{6}$, the OFDM symbol duration $T_{u}$ given by the DVB-T standard can be considered small enough to ensure that the phase rotation within one OFDM remains almost constant: $\mathrm{e}^{j 2 \pi \nu n \frac{T_{u}}{K}} \approx 1$ for all $n^{\prime} \in \mathcal{I}_{K}[15],[16]$. Hence, it is reasonable to make the following approximation simplifying the computation of each compensated signal block

$$
y_{\nu}^{m}[n] \approx y^{m}[n] \mathrm{e}^{-\jmath 2 \pi \nu m T_{s}}, \forall n \in \mathcal{I}_{K} .
$$

Based on the previous derivations, the detection scheme practical main steps can now be detailed as follows:

1) From the different blocks of the sampled surveillance signal, form the $M$ following vectors, $\forall m \in \mathcal{I}_{M}$ :

$$
\mathbf{y}^{m}=\left[y^{m}[0], \ldots, y^{m}[n], \ldots, y^{m}[K-1]\right]^{T} .
$$

2) Compute the $K$-points discrete Fourier transforms of each vector $\mathbf{y}^{m}$ :

$$
\mathbf{Y}^{m}=\left[Y^{m}[0], \ldots, Y^{m}[k], \ldots, Y^{m}[K-1]\right]^{T},
$$

with $\forall m \in \mathcal{I}_{M}, \forall k \in \mathcal{I}_{K}$ :

$$
Y^{m}[k]=\frac{1}{K} \sum_{n \in \mathcal{I}_{K}} y^{m}[n] \mathrm{e}^{-j 2 \pi \frac{k n}{K}} .
$$

3) Compute the $M$ channel frequency responses corresponding to the different blocks

$$
\mathbf{H}^{m}=\left[H^{m}[0], \ldots, H^{m}[k], \ldots, H^{m}[K-1]\right]^{T},
$$

with $\forall m \in \mathcal{I}_{M}$ :

$$
\begin{aligned}
& H^{m}[k]=Y^{m}[k] / d_{k}^{m}, \forall k \in \mathcal{E}_{K}, \\
& H^{m}[k]=0, \forall k \in \mathcal{I}_{K} \backslash \mathcal{E}_{K} .
\end{aligned}
$$

4) Choose a value $\nu$ for the Doppler compensation and compute the associated channel frequency response using the following linear combination (see (18) and (21))

$$
\hat{\mathbf{H}}_{\nu}=\frac{1}{M} \sum_{m \in \mathcal{I}_{M}} \mathrm{e}^{-j 2 \pi \nu m T_{s}} \mathbf{H}^{m} .
$$

5) Compute the detection function corresponding to the Doppler shift $\nu: \mathcal{D}(\tau, \nu)=\left|\hat{h}_{\nu}[n]\right|^{2}$, where $\hat{h}_{\nu}[n]$ is obtained by IDFT of vector $\hat{\mathbf{H}}_{\nu}$ (see (19)) and where $\tau=n T_{u} / K$.

6) Repeat steps 4) and 5) for different values of parameter $\nu$.

\section{Performance Analysis}

In this subsection, we compare for the two studied detectors (CAF and CHAD), the detection peak mean shape, and the floor mean levels for clutter and noise. The study will be conducted considering $d_{k}^{m}$ as a random variable and neglecting the effect of the pilot deterministic sequences inserted in the data stream.

Let us consider that the surveillance antenna is impinged by a single DVB-T signal $i$ whose coordinates are $\left(n_{i} \frac{T_{u}}{K}, f_{\mathrm{p}_{i}}\right)$ in the delay-Doppler plane. This signal should

${ }^{6}-500 \mathrm{~Hz}<\nu<500 \mathrm{~Hz}$ 
be seen as a potential source of disturbance (secondary lobes and a clutter floor) for the detection of a weaker target.

Assuming that the bistatic propagation delay $n_{i} \frac{T_{u}}{K}$ is less than the guard interval duration, an $m^{\text {th }}$ noise-free, powernormalized, received sampled block yields, $\forall n^{\prime} \in \mathcal{I}_{K}$

$$
y^{m}\left[n^{\prime}\right]=s^{m}\left[n^{\prime}-n_{i}\right] \mathrm{e}^{j 2 \pi f_{\mathrm{p}_{i}}\left(\frac{\left(n^{\prime}-n_{i}\right) T_{u}}{K}+m T_{s}\right)},
$$

where the unit-variance reference signal $s$ is assumed to be stationary block $m$ by block $m$ i.e. $\mathrm{E}\left\{\left|d_{k}^{m}\right|^{2}\right\}$ does not depend on $m$. Now since in the DVB-T standard, one has $\mathrm{E}\left\{\left|d_{k}^{m}\right|^{2}\right\}=1$, it comes that $\forall m$

$$
s^{m}\left[n^{\prime}\right]=\frac{1}{\sqrt{\# \mathcal{E}_{K}}} \sum_{k \in \mathcal{E}_{K}} d_{k}^{m} \mathrm{e}^{\jmath 2 \pi \frac{n^{\prime} k}{K}}
$$

ensuring the unit-variance assumption.

1) $C A F$ based detector: the cross-ambiguity function $\mathcal{A}$ is here defined as the squared modulus of the crosscorrelation function $\mathcal{X}$ :

$$
\mathcal{A}=|\mathcal{X}[n, \nu]|^{2},
$$

where

$$
\mathcal{X}[n, \nu]=\frac{1}{M K} \sum_{m \in \mathcal{I}_{M}} \sum_{n^{\prime} \in \mathcal{I}_{K}} y^{m}\left[n^{\prime}\right]\left(s^{m}\left[n^{\prime}-n\right]\right)^{*} \mathrm{e}^{-\jmath 2 \pi \nu m T_{s}},
$$

is computed from the symbol useful parts ( $M K$ samples) with a constant phase rotation within a symbol (see [14]). The two parameters under test $\left(n \frac{T_{u}}{K}, \nu\right)$ are respectively the bistatic propagation delay (bistatic range) and the Doppler-shifted frequency.

Using (23), the expression (26) yields

$$
\begin{aligned}
\mathcal{X}[n, \nu]= & \frac{\gamma}{M K} \sum_{m \in \mathcal{I}_{M}} \mathrm{e}^{\jmath 2 \pi\left(f_{\mathrm{p}_{i}}-\nu\right) m T_{s}} \\
& \sum_{n^{\prime} \in \mathcal{I}_{K}} s^{m}\left[n^{\prime}-n_{i}\right]\left(s^{m}\left[n^{\prime}-n\right]\right)^{*} \mathrm{e}^{\jmath 2 \pi f_{\mathrm{p}_{i}} \frac{n^{\prime} T_{u}}{K}},
\end{aligned}
$$

with $\gamma=\mathrm{e}^{-\jmath 2 \pi f_{\mathrm{p}_{i}} \frac{n_{i} T_{u}}{K}}$

Finally, replacing the expression of $s^{m}$ using (24), it comes

$$
\begin{aligned}
\mathcal{X}[n, \nu]= & \frac{\gamma}{M K \# \mathcal{E}_{K}} \sum_{m \in \mathcal{I}_{M}} \mathrm{e}^{2 \pi\left(f_{\mathrm{p}_{i}}-\nu\right) m T_{s}} \\
& \sum_{k, k^{\prime} \in \mathcal{E}_{K}^{2}} d_{k}^{m}\left(d_{k^{\prime}}^{m}\right)^{*} \mathrm{e}^{\frac{\jmath 2 \pi\left(n k^{\prime}-n_{i} k\right)}{K}} s_{k k^{\prime}}\left(f_{\mathrm{p}_{i}}\right),
\end{aligned}
$$

where

$$
\begin{aligned}
s_{k k^{\prime}}\left(f_{\mathrm{p}_{i}}\right) & \triangleq \sum_{n^{\prime} \in \mathcal{I}_{K}} \mathrm{e}^{\frac{j 2 \pi\left(k-k^{\prime}+f_{\left.\mathrm{p}_{i} T_{u}\right) n^{\prime}}\right.}{K}} \\
& =\frac{\sin \pi\left(k-k^{\prime}+f_{\mathrm{p}_{i}} T_{u}\right)}{\sin \pi\left(k-k^{\prime}+f_{\mathrm{p}_{i}} T_{u}\right) / K} .
\end{aligned}
$$

Note that $s_{k k}\left(f_{\mathrm{p}_{i}}\right)$ does not depend on $k$.

Now, observing a CAF detection output, for example in Figure 5, one can clearly distinguish, in the range-Doppler plane, both a non-stationary part and a stationary part. The non-stationary part (a squared sinc-like function) corresponds to the detection peak shape while the stationary part is a random variable we shall refer as the clutter floor. This behavior can be explained using the following decomposition:

$$
\mathcal{A}[n, \nu]=\left|\mathcal{X}^{\mathrm{e}}[n, \nu]+\mathcal{X}^{\mathrm{d}}[n, \nu]\right|^{2},
$$

with $\mathcal{X}^{\mathrm{e}}[n, \nu]=\mathcal{X}[n, \nu]$ when $k=k^{\prime}$ in (28),

$$
\text { and } \quad \mathcal{X}^{\mathrm{d}}[n, \nu]=\mathcal{X}[n, \nu] \text { when } k \neq k^{\prime} .
$$

Let us study the behavior of $\mathrm{E}\{\mathcal{A}[n, \nu]\}$. First, the random variables $d_{k}^{m}$ and $d_{k^{\prime}}^{m}$ being zero-mean and uncorrelated for all $k \neq k^{\prime}[17]$, it can easily be shown that $\mathcal{X}^{\mathrm{d}}[n, \nu]$ is centered for all $(n, \nu)$. Second, it is proved in Appendix $\mathrm{B}$ that $\mathcal{X}^{\mathrm{e}}$ and $\mathcal{X}^{\mathrm{d}}$ are uncorrelated. Then, introducing the zero-mean random function $\mathcal{Y} \triangleq \mathcal{X}^{\mathrm{e}}-\mathrm{E}\left\{\mathcal{X}^{\mathrm{e}}\right\}+\mathcal{X}^{\mathrm{d}}$, it comes

$$
\mathrm{E}\{\mathcal{A}[n, \nu]\}=\left|\mathrm{E}\left\{\mathcal{X}^{\mathrm{e}}\right\}\right|^{2}+\operatorname{Var}\{\mathcal{Y}\}
$$

As we will see subsequently, only the first right-hand side term of $(33)$ varies with $(n, \nu)$ : it corresponds to the peak shape; the second (constant) term matches the clutter floor level.

a) Peak shape: the CAF peak average shape is given by (see Appendix $\mathrm{C}$ for detailed derivations):

$\left|\mathrm{E}\left\{\mathcal{X}^{\mathrm{e}}[n, \nu]\right\}\right|^{2}=$

$\frac{\frac{\sin ^{2} \pi f_{\mathrm{p}_{i}} T_{u}}{\sin ^{2} \pi f_{\mathrm{p}_{i}} T_{u} / K}}{(M K)^{2}\left(\# \mathcal{E}_{K}\right)^{2}} \frac{\sin ^{2} \pi\left(f_{\mathrm{p}_{i}}-\nu\right) M T_{s}}{\sin ^{2} \pi\left(f_{\mathrm{p}_{i}}-\nu\right) T_{s}} \frac{\sin ^{2} \pi\left(n-n_{i}\right) \# \mathcal{E}_{K} / K}{\sin ^{2} \pi\left(n-n_{i}\right) / K}$.

As expected and due to the limited bandwidth, the peak shape corresponds to a squared sinc-like function (a Dirichlet kernel) limiting the resolution along the range axis. Similar secondary lobes are observed along the Doppler axis, depending on the number $M$ of OFDM symbols used.

Replacing $n$ by $n_{i}$ and $\nu$ by $f_{\mathrm{p}_{i}}$ in the latter equation and considering the approximation $\frac{\sin ^{2} \pi f_{\mathrm{p}_{i}} T_{u}}{\sin ^{2} \pi f_{\mathrm{p}_{i}} T_{u} / K} \approx K^{2}$, one obtains the detection peak mean level

$$
\left|\mathrm{E}\left\{\mathcal{X}^{\mathrm{e}}\left[n_{i}, f_{\mathrm{p}_{i}}\right]\right\}\right|^{2} \approx \frac{1}{M^{2}\left(\# \mathcal{E}_{K}\right)^{2}} M^{2}\left(\# \mathcal{E}_{K}\right)^{2} \quad \text { i.e. } 0 \mathrm{~dB} .
$$

b) Clutter floor mean level: we have

$$
\operatorname{Var}\{\mathcal{Y}\}=\operatorname{Var}\left\{\mathcal{X}^{\mathrm{e}}\right\}+\operatorname{Var}\left\{\mathcal{X}^{\mathrm{d}}\right\}
$$

The calculations of the variances of $\mathcal{X}^{\mathrm{e}}$ and $\mathcal{X}^{\mathrm{d}}$ are detailed respectively in Appendix D and Appendix E. We get

$$
\operatorname{Var}\left\{\mathcal{X}^{\mathrm{e}}[n, \nu]\right\}=\frac{\frac{\sin ^{2} \pi f_{\mathrm{p}_{i}} T_{u}}{\sin ^{2} \pi f_{\mathrm{p}_{i}} T_{u} / K}}{M K^{2} \# \mathcal{E}_{K}}\left(\mathrm{E}\left\{\left|d_{k}^{m}\right|^{4}\right\}-1\right)
$$


(Note that for QAM signals, $\mathrm{E}\left\{\left|d_{k}^{m}\right|^{4}\right\}$ never exceeds 1.381),

and

$$
\operatorname{Var}\left\{\mathcal{X}^{\mathrm{d}}\right\}=\frac{1}{M K^{2}\left(\# \mathcal{E}_{K}\right)^{2}} \sum_{k} \sum_{k^{\prime} \neq k}\left|s_{k k^{\prime}}\left(f_{\mathrm{p}_{i}}\right)\right|^{2} .
$$

It follows that $\operatorname{Var}\{\mathcal{Y}\}$ does not depend on $(n, \nu)$. Note that $\operatorname{Var}\left\{\mathcal{X}^{\mathrm{d}}\right\}<\operatorname{Var}\left\{\mathcal{X}^{\mathrm{e}}\right\}$ and the variable $\left|\mathcal{X}^{\mathrm{e}}[n, \nu]-\mathrm{E}\left\{\mathcal{X}^{\mathrm{e}}[n, \nu]\right\}\right|^{2}$ remains the main source of clutter.

c) Noise floor mean level: let us consider now that the received signal is white noise only: $y^{m}\left[n^{\prime}\right]=\eta^{m}\left[n^{\prime}\right]$. One can then write using (26)

$$
\begin{aligned}
\mathcal{X}[n, \nu]= & \frac{1}{M K \sqrt{\# \mathcal{E}_{K}}} \sum_{m \in \mathcal{I}_{M}} \mathrm{e}^{-\jmath 2 \pi \nu m T_{s}} \sum_{k \in \mathcal{E}_{K}}\left(d_{k}^{m}\right)^{*} \mathrm{e}^{\frac{j 2 \pi n k}{K}} \\
& \sum_{n^{\prime} \in \mathcal{I}_{K}} \eta^{m}\left[n^{\prime}\right] \mathrm{e}^{\frac{-\jmath 2 \pi n^{\prime} k}{K}} .
\end{aligned}
$$

Since the two independent random variables $d_{k}^{m}$ and $\eta^{m}\left[n^{\prime}\right]$ are assumed to be i.i.d. sequences, computing the modulus squared then the mathematical expectation, the noise floor mean level yields using $\mathrm{E}\left\{\left|d_{k}^{m}\right|^{2}\right\}=1$

$$
\begin{aligned}
\mathrm{E}\{\mathcal{A}[n, \nu]\} & =\frac{1}{M^{2} K^{2}} \sum_{m \in \mathcal{I}_{M}} \sum_{n^{\prime} \in \mathcal{I}_{K}} \mathrm{E}\left\{\left|\eta^{m}\left[n^{\prime}\right]\right|^{2}\right\} \\
& =\frac{\mathrm{E}\left\{\left|\eta^{m}\left[n^{\prime}\right]\right|^{2}\right\}}{M K} .
\end{aligned}
$$

2) CHAD: applying a DFT on a received symbol $y^{m}, Y^{m}\left[k^{\prime}\right] \triangleq \sum_{n^{\prime} \in \mathcal{I}_{K}} y^{m}\left[n^{\prime}\right] \mathrm{e}^{\frac{-j 2 \pi n^{\prime} k^{\prime}}{K}}$, and dividing by $\sqrt{\# \mathcal{E}_{K}} d_{k^{\prime}}^{m}, k^{\prime} \in \mathcal{E}_{K}$, one obtains after some derivations (see the Appendix F) the expression of the frequency response of the propagation channel corresponding to the reception of the $m^{t h}$ symbol:

$$
\begin{aligned}
& H^{m}\left[k^{\prime}\right] \triangleq \frac{Y^{m}\left[k^{\prime}\right]}{\sqrt{\# \mathcal{E}_{K}} d_{k^{\prime}}^{m}}= \\
& \frac{\gamma}{\# \mathcal{E}_{K}} \mathrm{e}^{\jmath 2 \pi f_{\mathrm{p}_{i}}\left(m T_{s}\right)} \sum_{k \in \mathcal{E}_{K}} \frac{d_{k}^{m}}{d_{k^{\prime}}^{m}} e^{\frac{-\jmath 2 \pi n_{i} k}{K}} s_{k k^{\prime}}\left(f_{\mathrm{p}_{i}}\right),
\end{aligned}
$$

where $\gamma=\mathrm{e}^{\frac{-\jmath 2 \pi f_{\mathrm{p}_{i}} n_{i} T_{u}}{K}}$.

Applying now the Doppler correction $\mathrm{e}^{-\jmath 2 \pi \nu\left(m T_{s}\right)}$ for each symbol $m$ and averaging over the $M$ available symbols, one has

$$
\begin{aligned}
& H_{\nu}\left[k^{\prime}\right] \triangleq \frac{1}{M} \sum_{m \in \mathcal{I}_{M}} \mathrm{e}^{-\jmath 2 \pi \nu\left(m T_{s}\right)} H^{m}\left[k^{\prime}\right]= \\
& \frac{\gamma}{M \# \mathcal{E}_{K}} \sum_{m \in \mathcal{I}_{M}} e^{\jmath 2 \pi\left(f_{\mathrm{p}_{i}}-\nu\right) m T_{s}} \sum_{k \in \mathcal{E}_{K}} \frac{d_{k}^{m}}{d_{k^{\prime}}^{m}} \mathrm{e}^{\frac{-\jmath 2 \pi n_{i} k}{K}} s_{k k^{\prime}}\left(f_{\mathrm{p}_{i}}\right) .
\end{aligned}
$$

The corresponding impulse response is obtained by IDFT:

$$
\begin{aligned}
h_{\nu}[n]= & \frac{\gamma}{M K \# \mathcal{E}_{K}} \sum_{m \in \mathcal{I}_{M}} \mathrm{e}^{j 2 \pi\left(f_{\mathrm{p}_{i}}-\nu\right) m T_{s}} \sum_{k^{\prime} \in \mathcal{E}_{K}} \mathrm{e}^{\frac{j 2 \pi n k^{\prime}}{K}} \\
& \sum_{k \in \mathcal{E}_{K}} \frac{d_{k}^{m}}{d_{k^{\prime}}^{m}} \mathrm{e}^{\frac{-j 2 \pi n_{i} k}{K}} s_{k k^{\prime}}\left(f_{\mathrm{p}_{i}}\right) .
\end{aligned}
$$

Let us consider the detection function

$$
\mathcal{D}[n, \nu]=\left|h_{\nu}[n]\right|^{2},
$$

with the following decomposition:

$$
h_{\nu}[n]=h_{\nu}^{\mathrm{e}}[n]+h_{\nu}^{\mathrm{d}}[n]
$$

where $h_{\nu}^{\mathrm{e}}$ gathers the terms in (44) with $k=k^{\prime}$ and where $h_{\nu}^{d}$ stands for the remaining terms $\left(k \neq k^{\prime}\right)$. Since $h_{\nu}^{\mathrm{e}}$ is deterministic (it does not depends on data $d_{k}^{m}$ ) and $h_{\nu}^{d}$ is a centered random variable (here $\mathrm{E}\left\{\frac{d_{k}^{m}}{d_{k^{\prime}}^{m}}\right\}=0$ ), it comes

$$
\mathrm{E}\{\mathcal{D}\}=\left|h_{\nu}^{\mathrm{e}}\right|^{2}+\mathrm{E}\left\{\left|h_{\nu}^{\mathrm{d}}\right|^{2}\right\}
$$

were the deterministic function $\left|h_{\nu}^{\mathrm{e}}\right|^{2}$ corresponds to the detection peak and the mean value of the random variable $\left|h_{\nu}^{\mathrm{d}}\right|^{2}$ corresponds to the clutter floor mean level.

a) Peak shape: let us study the deterministic part corresponding to the terms where $k=k^{\prime}$ in (44)

$$
\begin{aligned}
h_{\nu}^{\mathrm{e}}[n]= & \frac{\gamma}{M K \# \mathcal{E}_{K}} \sum_{m \in \mathcal{I}_{M}} \mathrm{e}^{j 2 \pi\left(f_{\mathrm{p}_{i}}-\nu\right) m T_{s}} \\
& \sum_{k \in \mathcal{E}_{K}} \mathrm{e}^{\frac{j 2 \pi\left(n-n_{i}\right) k}{K}} s_{k k}\left(f_{\mathrm{p}_{i}}\right) .
\end{aligned}
$$

Since $s_{k k}\left(f_{\mathrm{p}_{i}}\right)$ depends no more on $k$, it comes

$$
\begin{aligned}
& h_{\nu}^{\mathrm{e}}[n]= \\
& \frac{\gamma}{M K \# \mathcal{E}_{K}} \mathrm{e}^{\jmath \pi\left(f_{\mathrm{p}_{i}}-\nu\right)(M-1) T_{s}} \mathrm{e}^{\jmath \pi\left(n-n_{i}\right) \frac{K_{M}+K_{m}-1}{K}} \mathrm{e}^{\jmath \pi f_{\mathrm{p}_{i}} T_{u} \frac{K-1}{K}} \\
& \frac{\sin \pi\left(f_{\mathrm{p}_{i}}-\nu\right) M T_{s}}{\sin \pi\left(f_{\mathrm{p}_{i}}-\nu\right) T_{s}} \frac{\sin \pi\left(n-n_{i}\right) \# \mathcal{E}_{K} / K}{\sin \pi\left(n-n_{i}\right) / K} \frac{\sin \pi f_{\mathrm{p}_{i}} T_{u}}{\sin \pi f_{\mathrm{p}_{i}} T_{u} / K} .
\end{aligned}
$$

Using $|\gamma|=1$, we straightforwardly obtain the CHAD detection peak shape as the squared modulus of the latter expression:

$$
\begin{aligned}
\left|h_{\nu}^{\mathrm{e}}[n]\right|^{2}= & \frac{\frac{\sin ^{2} \pi f_{\mathrm{p}_{i}} T_{u}}{\sin ^{2} \pi f_{\mathrm{p}_{i}} T_{u} / K}}{M^{2} K^{2}\left(\# \mathcal{E}_{K}\right)^{2}} \frac{\sin ^{2} \pi\left(n-n_{i}\right) \# \mathcal{E}_{K} / K}{\sin ^{2} \pi\left(n-n_{i}\right) / K} \\
& \frac{\sin ^{2} \pi\left(f_{\mathrm{p}_{i}}-\nu\right) M T_{s}}{\sin ^{2} \pi\left(f_{\mathrm{p}_{i}}-\nu\right) T_{s}} .
\end{aligned}
$$

which appear identical to that obtained with classical CAF.

b) Clutter floor mean level: as for CAF, the clutter forms a stochastic pedestal. The difference is that, here, only the terms where $k \neq k^{\prime}$ contribute to the clutter floor. 
Using (44), one finds:

$$
\mathrm{E}\left\{\left|h_{\nu}^{\mathrm{d}}[n]\right|^{2}\right\}=\frac{1}{\left(M K \# \mathcal{E}_{K}\right)^{2}} \sum_{m, m^{\prime} \in \mathcal{I}_{M}^{2}} \mathrm{e}^{\jmath 2 \pi\left(f_{\mathrm{p}_{i}}-\nu\right)\left(m-m^{\prime}\right) T_{s}}
$$

$$
\begin{aligned}
& \sum_{k_{2}, k_{2}^{\prime} \in \mathcal{E}_{K}^{2}} \mathrm{e}^{\frac{j 2 \pi n\left(k_{2}-k_{2}^{\prime}\right)}{K}} \sum_{k_{1} \neq k_{2}, k_{1}^{\prime} \neq k_{2}^{\prime} \in \mathcal{E}_{K}^{2}} \mathrm{E}\left\{\frac{d_{k_{1}}^{m}}{d_{k_{2}}^{m}} \frac{\left(d_{k_{1}^{\prime}}^{m^{\prime}}\right)^{*}}{\left(d_{k_{2}^{\prime}}^{m)^{\prime}}\right)^{*}}\right\} \\
& \mathrm{e}^{\frac{j 2 \pi n_{i}\left(k_{1}^{\prime}-k_{1}\right)}{K}} s_{k_{1} k_{2}}\left(f_{\mathrm{p}_{i}}\right) s_{k_{1}^{\prime} k_{2}^{\prime}}^{*}\left(f_{\mathrm{p}_{i}}\right) .
\end{aligned}
$$

Since $\mathrm{E}\left\{d_{k}^{m}\right\}=0$ and $\mathrm{E}\left\{\frac{1}{d_{k}^{m}}\right\}=0$, then $\mathrm{E}\left\{\frac{d_{k_{1}}^{m}}{d_{k_{2}}^{m}} \frac{\left(d_{k_{1}^{\prime}}^{m}\right)^{*}}{\left(d_{k_{2}^{\prime}}^{m \prime^{\prime}}\right)^{*}}\right\}=$ 0 for $m \neq m^{\prime}$. Additionally, it can be shown that for QAM signals $\mathrm{E}\left\{\frac{d_{k}^{m}}{\left(d_{k}^{m}\right)^{*}}\right\}=0$, hence only the terms with $k_{1}=k_{1}^{\prime}$ and $k_{2}=k_{2}^{\prime}$ are not null in the previous expression and the clutter mean level yields

$$
\operatorname{Var}\left\{h_{\nu}^{\mathrm{d}}[n]\right\}=\frac{\mathrm{E}\left\{\frac{1}{\left|d_{k}^{m}\right|^{2}}\right\}}{M\left(K \# \mathcal{E}_{K}\right)^{2}} \sum_{k} \sum_{k^{\prime} \neq k} s_{k k^{\prime}}^{2}\left(f_{\mathrm{p}_{i}}\right) .
$$

c) Noise floor mean level: as for CAF, let us consider that the received signal is white noise only: $y^{m}\left[n^{\prime}\right]=$ $\eta^{m}\left[n^{\prime}\right]$. One obtains the following Doppler channel frequency response

$$
\begin{aligned}
H_{\nu}[k]= & \frac{1}{M} \sum_{m \in \mathcal{I}_{M}} \frac{Y^{m}[k]}{\sqrt{\# \mathcal{E}_{K}} d_{k}^{m}} \mathrm{e}^{-\jmath 2 \pi \nu m T_{s}} \\
= & \frac{1}{M \sqrt{\# \mathcal{E}_{K}}} \sum_{m \in \mathcal{I}_{M}} \frac{1}{d_{k}^{m}} \mathrm{e}^{-\jmath 2 \pi \nu m T_{s}} \\
& \sum_{n^{\prime} \in \mathcal{I}_{K}} \eta^{m}\left[n^{\prime}\right] \mathrm{e}^{\frac{-22 \pi n^{\prime} k}{K}} .
\end{aligned}
$$

Applying a IDFT, we get the corresponding impulse response

$$
\begin{aligned}
h_{\nu}[n]= & \frac{1}{M K \sqrt{\# \mathcal{E}_{K}}} \sum_{k \in \mathcal{E}_{K}} \sum_{m \in \mathcal{I}_{M}} \frac{1}{d_{k}^{m}} \mathrm{e}^{-\jmath 2 \pi \nu m T_{s}} \\
& \sum_{n^{\prime} \in \mathcal{I}_{K}} \eta^{m}\left[n^{\prime}\right] \mathrm{e}^{\frac{j 2 \pi\left(n-n^{\prime}\right) k}{K}} .
\end{aligned}
$$

The two independent random variables $d_{k}^{m}$ and $\eta^{m}\left[n^{\prime}\right]$ being zero mean and temporally white, variance of $h_{\nu}[n]$ yields

$$
\begin{aligned}
\mathrm{E}\left\{\left|h_{\nu}[n]\right|^{2}\right\}= & \frac{1}{(M K)^{2} \# \mathcal{E}_{K}} \sum_{m \in \mathcal{I}_{M}} \sum_{k \in \mathcal{E}_{K}} \mathrm{E}\left\{\frac{1}{\left|d_{k}^{m}\right|^{2}}\right\} \\
& \sum_{n^{\prime} \in \mathcal{I}_{K}} \mathrm{E}\left\{\left|\eta^{m}\left[n^{\prime}\right]\right|^{2}\right\} \\
= & \rho \frac{\mathrm{E}\left\{\left|\eta^{m}\left[n^{\prime}\right]\right|^{2}\right\}}{M K}
\end{aligned}
$$

where $\rho=\frac{1}{\# \mathcal{E}_{K}} \sum_{k \in \mathcal{E}_{K}} \mathrm{E}\left\{\frac{1}{\left|d_{k}^{m}\right|^{2}}\right\} \approx \mathrm{E}\left\{\frac{1}{\left|d_{k}^{m}\right|^{2}}\right\}$.

This latter expression indicates that the noise floor mean level depends on the QAM type. On can note that for 4-QAM, CAF and CHAD exhibits the same noise floor level $(\rho=1)$. For other types of QAM, the noise floor is unfortunately higher, since one has $\rho=1.8$ for $16-\mathrm{QAM}(+2.5 \mathrm{~dB})$, and $\rho=2.6$ for $64-\mathrm{QAM}(+4.1 \mathrm{~dB})$.

\section{Performance comparison}

From the clutter floor theoretical mean level of each studied detector (37) for CAF and (51) for CHAD, we will evaluate the improvement brought by CHAD compared to CAF.

Let us consider the two zero mean complex variables: $\mathcal{Y}=\mathcal{X}^{\mathrm{e}}-\mathrm{E}\left\{\mathcal{X}^{\mathrm{e}}\right\}+\mathcal{X}^{\mathrm{d}}$ (for CAF) and $\mathcal{Z} \triangleq h_{\nu}^{\mathrm{d}}$ (for CHAD), and the clutter floor attenuation coefficient defined by $c_{\mathcal{Z} / \mathcal{Y}}\left(f_{\mathrm{p}_{i}}\right) \triangleq \frac{\operatorname{Var}\{\mathcal{Z}\}}{\operatorname{Var}\{\mathcal{Y}\}}$

The attenuation $c_{\mathcal{Z} / \mathcal{Y}}\left(f_{\mathrm{p}_{i}}\right)$, depends on the absolute value of the Doppler-shifted frequency $f_{\mathrm{p}_{i}}$ of the disturbance source $\left(s_{k k^{\prime}}\left(f_{\mathrm{p}_{i}}\right)\right.$ is an even function) and is parametrized by the number of subcarriers and the QAM type. One has after some developments:

$$
\begin{gathered}
c_{\mathcal{Z} / \mathcal{Y}}\left(f_{\mathrm{p}_{i}}\right)=\frac{\operatorname{Var}\left\{h_{\nu}^{d}\right\}}{\operatorname{Var}\{\mathcal{X} \mathrm{e}\}+\operatorname{Var}\left\{\mathcal{X}^{\mathrm{d}}\right\}}= \\
\frac{\mathrm{E}\left\{\frac{1}{\left|d_{k}^{m}\right|^{2}}\right\} \sum_{k} \sum_{k^{\prime} \neq k} s_{k k^{\prime}}^{2}\left(f_{\mathrm{p}_{i}}\right)}{\frac{\# \mathcal{E}_{K} \sin ^{2} \pi f_{\mathrm{p}_{i}} T_{u}}{\sin ^{2} \pi f_{\mathrm{p}_{i}} T_{u} / K}\left(\mathrm{E}\left\{\left|d_{k}^{m}\right|^{4}\right\}-1\right)+\sum_{k} \sum_{k^{\prime} \neq k} s_{k k^{\prime}}^{2}\left(f_{\mathrm{p}_{i}}\right)} .
\end{gathered}
$$

Figure 2 presents plots of the attenuation $c_{\mathcal{Z} / \mathcal{Y}}$ according to the disturbing path Doppler shift $f_{\mathrm{p}_{i}}$, for 64-QAM with the two DVB-T $K$-modes.

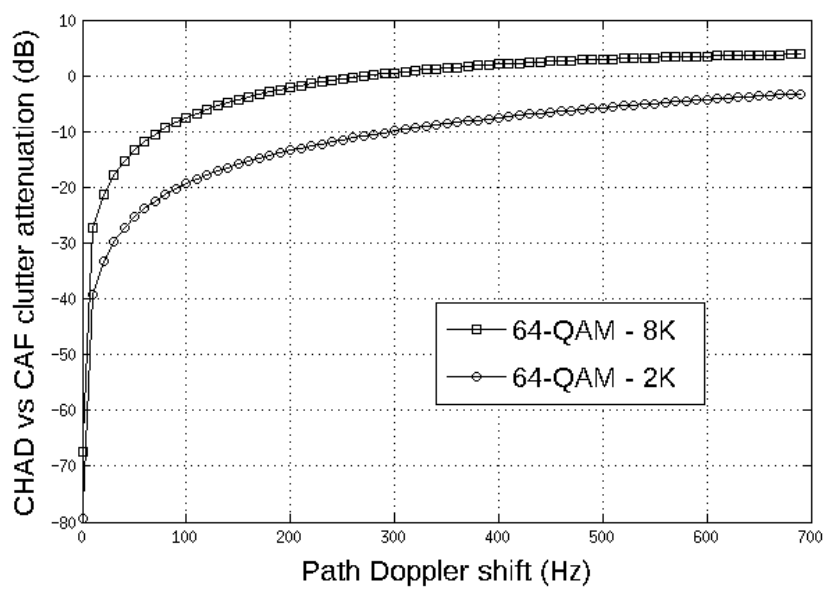

Fig. 2. CHAD/CAF clutter floor attenuation coefficient $c_{\mathcal{Z} / \mathcal{Y}}(\mathrm{dB})$.

One can see that, using CHAD, the clutter floor level remains lower than that obtained with a CAF for low frequencies (up to about $300 \mathrm{~Hz}$ for the $8 \mathrm{~K}$ mode). In particular and very interestingly, since $s_{k k^{\prime}}(0)=0$, the clutter floor induced by the set of zero-Doppler paths reduces to zero.

The variables $\mathcal{Y}(\mathrm{CAF})$ and $\mathcal{Z}(\mathrm{CHAD})$ are obtained by summing a large number $(M K)$ of independent data $d_{k}^{m}$. Hence, invoking the central limit 
theorem, it can be considered that these variables are normally distributed. Moreover, since $\operatorname{Re}\left\{d_{k}^{m}\right\}$ and $\operatorname{Im}\left\{d_{k}^{m}\right\}$ are independent random variables with the same variance, one has for the two following real random vectors: $(\operatorname{Re}\{\mathcal{Y}\}, \operatorname{Im}\{\mathcal{Y}\}) \sim \mathcal{N}\left(0, \sqrt{\frac{\operatorname{Var}\{\mathcal{Y}\}}{2}} \mathbf{I}_{2}\right)$ and $(\operatorname{Re}\{\mathcal{Z}\}, \operatorname{Im}\{\mathcal{Z}\}) \sim \mathcal{N}\left(0, \sqrt{\frac{\operatorname{Var}\{\mathcal{Z}\}}{2}} \mathbf{I}_{2}\right)$. The two variables $\frac{2}{\operatorname{Var}\{\mathcal{Y}\}}|\mathcal{Y}|^{2}$ and $\frac{2}{\operatorname{Var}\{\mathcal{Z}\}}|\mathcal{Z}|^{2}$ are then chi-squared distributed with 2 degrees of freedom $\left(\chi_{2}^{2}\right)$ and we get the two following results

$$
\begin{aligned}
& \operatorname{Prob}\left\{|\mathcal{Y}|^{2}>\frac{9.21 \operatorname{Var}\{\mathcal{Y}\}}{2}\right\}=1 \%, \\
& \operatorname{Prob}\left\{|\mathcal{Z}|^{2}>\frac{9.21 \operatorname{Var}\{\mathcal{Z}\}}{2}\right\}=1 \%,
\end{aligned}
$$

giving a $1 \%$ confidence interval for the clutter floor level of each detector.

As an example, Figure 3 presents, for a 64-QAM - 8K, 90 symbols emission, the clutter floor $1 \%$ confidence intervals (in $\mathrm{dB}$ ) for both CAF and CHAD.

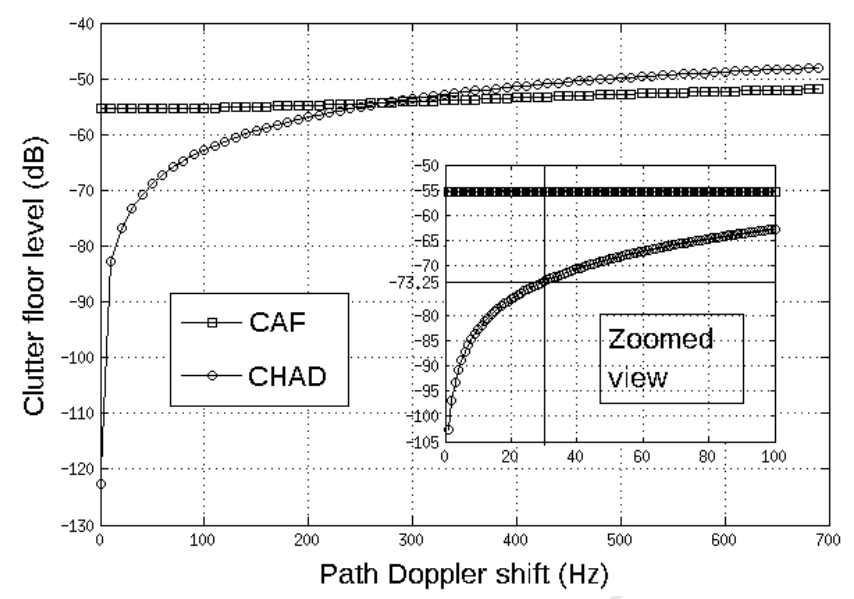

Fig. 3. Clutter floor level for a unit-power source, 90 symbols, 64-QAM, $8 \mathrm{~K}$ (1\% confidence interval in $\mathrm{dB}$ ).

\section{E. Short study of the case: $\tau_{i}>T_{c p}$}

Without any loss of generality, we consider a single delayed static contribution, such that $T_{c p}<\tau_{i}<T_{u}$ ( i.e $\left.0<n_{i}<K-1\right)$. It follows an overlapping of symbol $m+1$ on the $m^{\text {th }}$ received block. For a noise-free case, one has from Equation (14)

$$
\begin{aligned}
y^{m}\left[n^{\prime}\right] & =\sum_{k^{\prime} \in \mathcal{E}_{K}} H_{0}\left[k^{\prime}\right] d_{k^{\prime}}^{m-1} \mathrm{e}^{j 2 \pi \frac{k^{\prime} n}{K}} \quad n^{\prime}=0, \ldots, n_{i}-1 \\
& +\sum_{k^{\prime} \in \mathcal{E}_{K}} H_{0}\left[k^{\prime}\right] d_{k^{\prime}}^{m} \mathrm{e}^{j 2 \pi \frac{k^{\prime} n^{\prime}}{K}} \quad n^{\prime}=n_{i}, \ldots, K-1
\end{aligned}
$$

After DFT and data division, we obtain

$$
\begin{aligned}
\frac{Y^{m}[k]}{d_{k}^{m}} & =\frac{1}{K} \sum_{n^{\prime}=0}^{K-1} \frac{y^{m}\left[n^{\prime}\right]}{d_{k}^{m}} \mathrm{e}^{-j 2 \pi \frac{k n^{\prime}}{K}} \\
& =\frac{1}{K} \sum_{n^{\prime}=0}^{n_{i}-1} \sum_{k^{\prime} \in \mathcal{E}_{K}} H_{0}\left[k^{\prime}\right] \frac{d_{k^{\prime}}^{m-1}}{d_{k}^{m}} \mathrm{e}^{j 2 \pi \frac{\left(k^{\prime}-k\right) n^{\prime}}{K}} \\
& +\frac{1}{K} \sum_{n^{\prime}=n_{i}}^{K-1} \sum_{k^{\prime} \in \mathcal{E}_{K}} H_{0}\left[k^{\prime}\right] \frac{d_{k^{\prime}}^{m}}{d_{k}^{m}} \mathrm{e}^{j 2 \pi \frac{\left(k^{\prime}-k\right) n^{\prime}}{K}}
\end{aligned}
$$

whose the expectation value is

$$
\begin{aligned}
\mathrm{E}\left\{\frac{Y^{m}[k]}{d_{k}^{m}}\right\} & =\frac{1}{K} \sum_{n^{\prime}=0}^{n_{i}-1} \sum_{k^{\prime} \in \mathcal{E}_{K}} H_{0}\left[k^{\prime}\right] \mathrm{E}\left\{\frac{d_{k^{\prime}}^{m-1}}{d_{k}^{m}}\right\} \mathrm{e}^{j 2 \pi \frac{\left(k^{\prime}-k\right) n^{\prime}}{K}} \\
& +\frac{1}{K} \sum_{n^{\prime}=n_{i}}^{K-1} \sum_{k^{\prime} \in \mathcal{E}_{K}} H_{0}\left[k^{\prime}\right] \mathrm{E}\left\{\frac{d_{k^{\prime}}^{m}}{d_{k}^{m}}\right\} \mathrm{e}^{j 2 \pi \frac{\left(k^{\prime}-k\right) n^{\prime}}{K}} \\
& =\frac{1}{K} \sum_{n^{\prime}=n_{i}}^{K-1} H_{0}[k]=\frac{K-n_{i}}{K} H_{0}[k] .
\end{aligned}
$$

Thus, when $\tau_{i}$ varies from $T_{c p}$ to $T_{s}$, the corresponding channel frequency response linearly decreases. When $\tau_{i}>T_{s}$, it remains null. This result is still valid for any Doppler echo.

\section{ZDC REJECTION FROM STATIC CHANNEL ESTIMATION}

Whether with CHAD or CAF, the static echo secondary lobes remain and their strong amplitude can still compromise the detection of the weakest targets. This disturbing zero-Doppler contribution has then to be removed and we therefore propose two ways.

\section{A. ZDC rejection preprocessing}

A classical strategy is to implement the $\mathrm{ZDC}$ rejection as a preprocessing basically applied on the received signal $y[k]$ (see e.g. [6], [8], [9]). As in [9], it can be done here using the estimation $\hat{H}_{0}[k]$ of the zero-Doppler channel frequency response (obtained using (18) with $\nu=0$ ). Then, we directly get the estimate of the spectral representation of the dynamic part, computing for each block $m \in \mathcal{I}_{M}$ :

$$
\tilde{Y}^{m}[k] \simeq Y^{m}[k]-\hat{H}_{0}[k] d_{k}^{m}, \quad k \in \mathcal{E}_{K} .
$$

This ZDC-free signal can be used to detect most of the formerly hidden targets, implementing a classical CAF or a CHAD detector.

Note that, similarly to the approach in [8], the computational cost (the number of complex multiplications) of such a preprocessing is only $\mathcal{O}\left(M K \log _{2}(K)\right)$ while the least mean squares (LMS) method [6] exhibits a complexity having a cubic growth with $K\left(\mathcal{O}\left(M K^{3}\right)\right)$. 


\section{B. Online $Z D C$ rejection}

The CHAD approach allows to directly implement the ZDC rejection during the estimation of the Doppler channel frequency response. From (62), it can be done replacing (22) by

$$
\hat{\mathbf{H}}_{\nu}=\frac{1}{M} \sum_{m}\left(\mathbf{H}^{m}-\hat{\mathbf{H}}_{0}\right) \mathrm{e}^{-\jmath 2 \pi \nu m T_{s}},
$$

where $\hat{\mathbf{H}}_{0}=\frac{1}{M} \sum_{m} \mathbf{H}^{m}$ corresponds to the static channel vector estimation and where $\hat{\mathbf{H}}_{\nu}$ stands now for the ZDCfree stationarized Doppler channel. After some derivations, it can be rewritten as

$$
\hat{\mathbf{H}}_{\nu}=\frac{1}{M} \sum_{m}\left(\mathrm{e}^{-\jmath 2 \pi \nu m T_{s}}-\frac{1-\mathrm{e}^{-\jmath 2 \pi \nu M T_{s}}}{M\left(1-\mathrm{e}^{\left.-\jmath 2 \pi \nu T_{s}\right)}\right.}\right) \mathbf{H}^{m},
$$

unifying in one and the same step the ZDC rejection and the detector construction while not increasing the computational complexity.

\section{CHAD ALGORITHM}

Based on the previous derivations, the resulting channel detector algorithm (CHAD) is given in Table I.

TABLE I

CHAD (Channel Detector)

$$
\begin{aligned}
& \text { 0) Inputs } \\
& \mathbf{d}^{m}=\left[d_{0}^{m}, \ldots, d_{k}^{m}, \ldots, d_{K-1}^{m}\right]^{T}, m \in \mathcal{I}_{M} \\
& \mathbf{y}^{m}=\left[y^{m}[1], \ldots, y^{m}\left[n^{\prime}\right], \ldots, y^{m}[K-1]\right]^{T}, m \in \mathcal{I}_{M} \\
& \text { 1) Compute } \mathbf{H}^{m}=\operatorname{DFT}\left[\mathbf{y}^{m}\right] \odot \frac{1}{\mathbf{d}^{m}} \\
& \text { where } \odot \text { stands for the element-wise product } \\
& \text { 2) Estimation of the Doppler channel impulse responses } \\
& \text { for } l=-L / 2, \ldots, L / 2 \\
& \begin{array}{ll}
\text { a. } & \nu[l]=l \Delta_{\nu} \\
\text { b. } & \mathbf{H}_{\nu}=\frac{1}{M} \sum_{m} \mathbf{H}^{m} w_{m},
\end{array} \\
& w_{m}= \begin{cases}\mathrm{e}^{-\jmath 2 \pi \nu m T_{s}} \text { for a straightforward detection } \\
\mathrm{e}^{-\jmath 2 \pi \nu m T_{s}}-\frac{1-\mathrm{e}^{-\jmath 2 \pi \nu M T_{s}}}{M\left(1-\mathrm{e}^{-\jmath 2 \pi \nu T_{s}}\right)} & \text { detection including } \\
& \text { ZDCjection }\end{cases} \\
& \text { c. } \mathbf{h}_{\nu}=\operatorname{IDFT}\left[\mathbf{H}_{\nu}\right] \\
& \text { end } \\
& \text { 3) Echo detection } \\
& \text { Construct the } K \times L \text { detection map } \mathcal{D} \text { such that } \mathcal{D}[n, l]= \\
& \left|h_{\nu[l]}[n]\right|^{2} \text {. }
\end{aligned}
$$

Note that since $\frac{1}{d_{k}^{m}}=\frac{\left(d_{k}^{m}\right)^{*}}{\left|d_{k}^{m}\right|^{2}}$, we retrieve here the approach in [13] where the reference signal is mismatched by dividing by the squared modulus of each datum $d_{k}^{m}$ (modulus equalization). As outlined in section I, Searle et al. [13] propose to ignore the secondary lobes by computing the "mismatched reference signal" CAF with a Doppler shift resolution such that "any Fourier bin exists at a zero of any other bin", that is to say using $\Delta_{\nu}=\frac{1}{M T_{s}}$. It is very interesting because ZDC sidelobes (artificially) disappear and ZDC mitigation may appear now useless. However, it follows a detection loss and consequently we preferred to keep in most of the numerical results, both an oversampling on the Doppler dimension and a ZDC cancellation. Note that as suggested in [14], [13], an ad hoc Doppler shift range allows the straightforward estimate of $\mathbf{H}_{\nu}$ by FFT.

\section{Numerical Simulations}

We consider a simulation scenario where four moving targets are illuminated by a 8K, 64-QAM, DVB$\mathrm{T}$ emission with $T_{c p}=T_{u} / 16$. The target echoes $\mathrm{Tg}_{1}, \mathrm{Tg}_{2}, \mathrm{Tg}_{3}$ and $\mathrm{Tg}_{4}$ are received with respectively a $40 \mathrm{~dB}, 60 \mathrm{~dB}, 65 \mathrm{~dB}$ and $90 \mathrm{~dB}$ power attenuation (relatively to the LOS emission). Their coordinates in the range-frequency plane are respectively: $(3 \mathrm{~km}, 30 \mathrm{~Hz})$, $(6 \mathrm{~km}, 90 \mathrm{~Hz}),(7 \mathrm{~km},-150 \mathrm{~Hz})$ and $(12 \mathrm{~km},-100 \mathrm{~Hz})$. Additionally, we simulate the presence of about 30 static echoes with a minimal attenuation of $30 \mathrm{~dB}$. These echoes are randomly distributed in a bistatic range less than 16 $\mathrm{km}$ and have consequently bistatic propagation delays less than the guard interval. A $50 \mathrm{~dB}$ attenuation AWGN channel is considered. Finally, the Doppler shift step $\Delta_{\nu}$ is set to $1 \mathrm{~Hz}$.

Figure 4 presents a CAF output computed over 90 consecutive OFDM symbols (i.e. a $85 \mathrm{~ms}$ duration and $90 \times 8192=737280$ samples) from the original received sequence $(y[n])_{n \in \mathcal{I}_{N}}$.

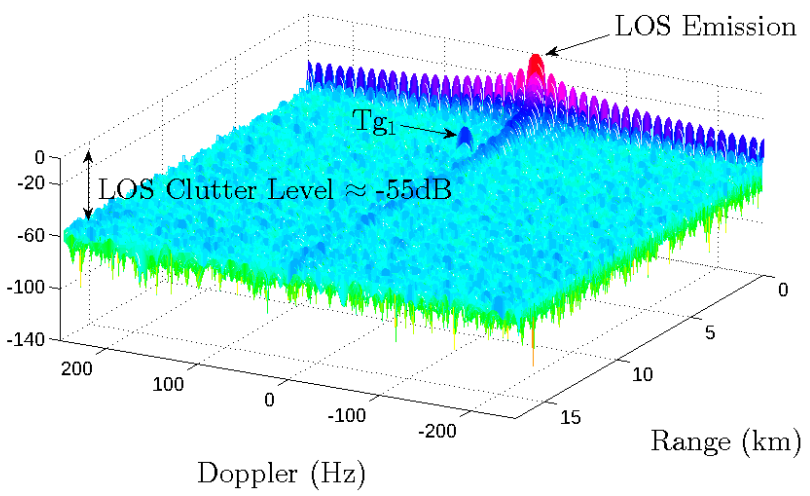

Fig. 4. CAF using the original received signal.

The stochastic pedestal level induced by the LOS corresponding peak does not exceed $(1 \%$ confidence interval) about -55 dB (see Figure 3 for a null Dopplershifted frequency). Consequently, and as it can be seen in the figure, only the most powerful target $\mathrm{Tg}_{1}$ remains visible while the other three Doppler echoes are hidden by the LOS clutter level.

Three ZDC rejection preprocessings have been implemented and tested before the CAF detection. Figure 5 shows a CAF output obtained using the approach proposed in subsection IV-A . 


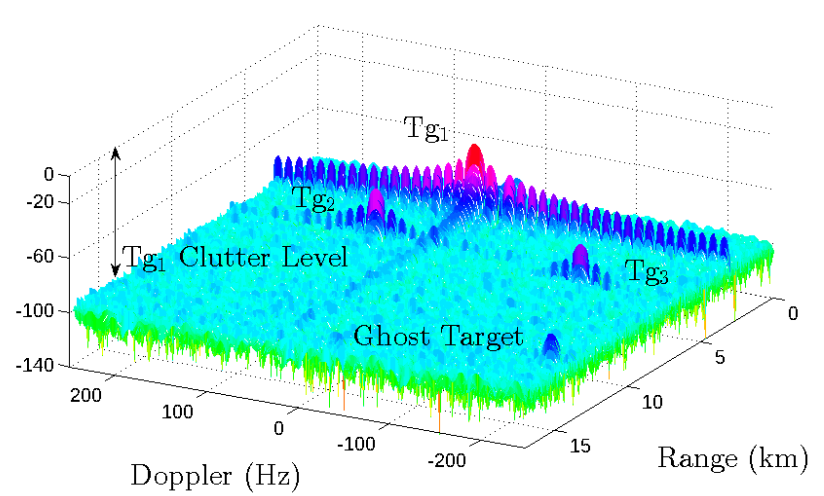

Fig. 5. CAF after a ZDC rejection prior stage.

The stochastic floor level induced by the new dominant echo $\operatorname{Tg}_{1}(30 \mathrm{~Hz})$ reduces to $-95 \mathrm{~dB}$, corresponding to the theoretical value obtained by combining its power attenuation $(-40 \mathrm{~dB})$ and the CAF clutter level for a unitpower source $(-55 \mathrm{~dB}$, see the zoomed view in Figure 3). The targets $\mathrm{Tg}_{2}$ and $\mathrm{Tg}_{3}$ are now detected, but $\mathrm{Tg}_{4}$ outcrops the clutter level and is then not clearly visible. A false detection peak betraying the presence of pilot signals, appears (ghost target on the figure). Note that this ambiguity peak could be reduced using a reference signal synthesized with a pilot level reduction [10], [11].

Very close results are obtained by the other two preprocessings we tested: the LMS-based method [6] and the zero-Doppler orthogonal projection [8]. For the LMS-based method, both a high computational load $\left(\mathcal{O}\left(M K^{3}\right)\right)$, and a high memory load are necessary since a $M K \times T_{c p}=M K \times K / 16$ dimensional matrix has to be pseudo-inverted.

Figure 6 presents the detection map obtained using CHAD (90 symbols) without ZDC rejection (22).

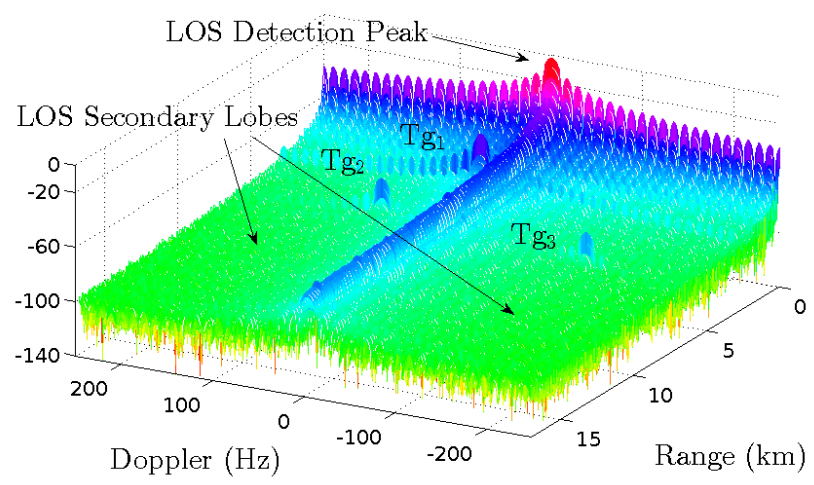

Fig. 6. CHAD output $\mathcal{D}$ without $\mathrm{ZDC}$ rejection.

Since the stochastic pedestal induced by the static emissions is now null, the three most powerful targets are clearly detected but the weakest target remains hidden by the secondary lobes of the LOS detection peak. Finally, one can see that, since CHAD detection peak is data-independent (see eq. (49)), the ghost peak appears no more.

Figure 7 corresponds to the detection map obtained with CHAD using an $H_{0}$ compensation (64) in order to remove the LOS secondary lobes. The clutter level induced by the most powerful remaining target $\mathrm{Tg}_{1}$ is $-113.25 \mathrm{~dB}$ (obtained by combining the $-40 \mathrm{~dB}$ power attenuation and the $-73.25 \mathrm{~dB}$ CHAD theoretical clutter level of Figure 3), that is to say less than the noise floor $\left(-50-10 \log _{10}(90 \times 8192) \approx-108 \mathrm{~dB}\right)$ which becomes the main hiding effect.

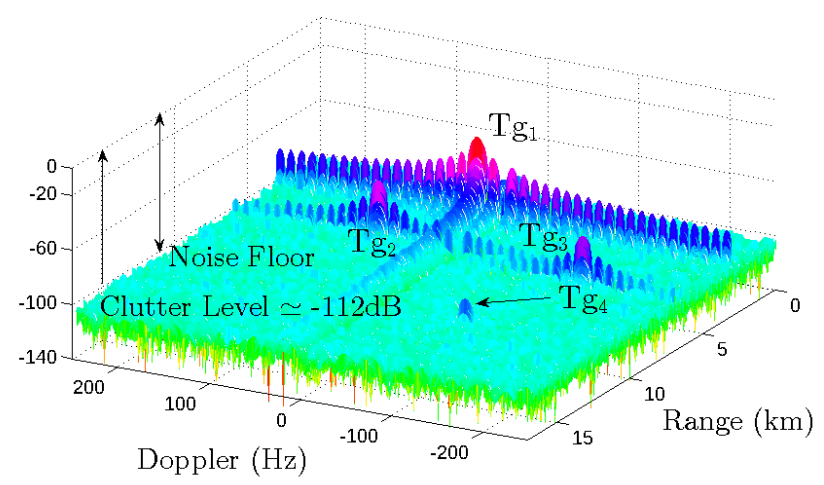

Fig. 7. CHAD output $\mathcal{D}$ including $H_{0}$ compensation.

The weakest target $\operatorname{Tg}_{4}$ is now detected.

\section{EXPERIMENTAL DATA}

We present here a detection result obtained with an acquisition system located near the city of Rennes in France. The target is a ferryboat illuminated by the DVB$\mathrm{T}$ transmitter "Bécherel" whose modulation parameters are: $8 \mathrm{~K}$ mode, 64 -QAM and $T_{c p}=T_{u} / 32$. In the rangefrequency plane, the target bistatic coordinates are about (46 km, $-67 \mathrm{~Hz})$.

The figure 8 presents the detection maps obtained without ZDC rejection with both $\mathrm{CAF}$ (left) and CHAD (right) using $M=190$ OFDM symbols and $\Delta_{\nu}=1 \mathrm{~Hz}$.

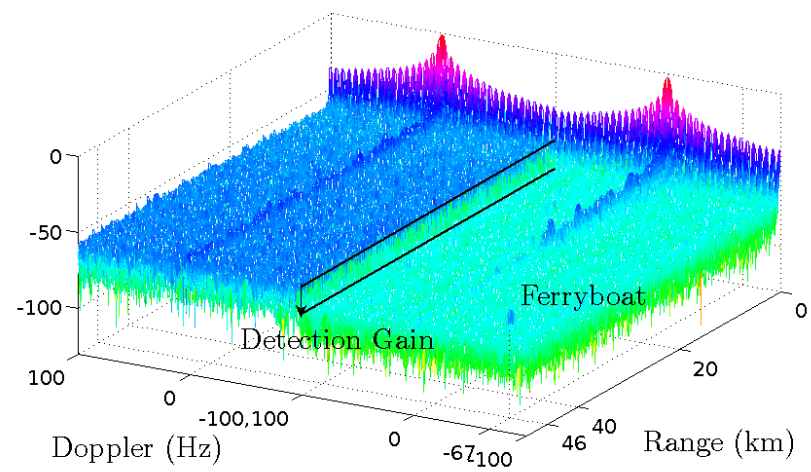

Fig. 8. CAF (left) and CHAD (right) on real data without ZDC rejection. 
The ferryboat location conducts to a bistatic delay higher than six times the CP duration and consequently out of the $\tau_{i}<T_{c p}$ assumption. It follows (see III-E) a detection loss depending on the amount of data outside the guard interval i.e. here about $10 \log _{10}\left((32-6)^{2} / 32^{2}\right) \approx-1.8 \mathrm{~dB}$, see eq. (61). One can see that despite this slight loss of detection, the clutter level being greatly attenuated (about $30 \mathrm{~dB}$ ), the target is clearly visible (lower right corner) with CHAD while classical CAF fails.

Note that the clutter reduction level obtained with CHAD is apparently lower than in the previous simulations. This can be explained by a higher noise floor which is reached here, limiting the performance.

The last figure 9 presents the detection maps obtained by each detector after a ZDC rejection.



Fig. 9. CAF (left) and CHAD (right) on real data with ZDC rejection.

The results are close to each other but one can observe that, as expected, CHAD exhibits a noise floor level slightly higher than the classical CAF.

\section{ViII. Conclusion and Perspectives}

In this paper, we introduce the notion of "Doppler channel". We show how its associated impulse response can be efficiently computed for DVB-T emissions and used as a new performing target detection tool, we named CHAD (channel detector). We theoretically compare the performance of CHAD to that of classical CAF. In particular, we demonstrate that, very interestingly, for low Doppler-shifted contributions, the induced clutter level remains significantly inferior to that of CAF. This level even reaches zero for zero-Doppler contributions.

CHAD actually appears to be equivalent to a CAF based on the mismatched reference signal proposed in [12], [13]. However, this new interpretation in terms of Doppler channels opens a much wider range of possibilities. For example, one can now directly merge the ZDC rejection with the detector without increasing the computational load. In the Doppler channel domain, the detection problem becomes a simple harmonic analysis problem and one can imagine that we might improve the performance, using e.g. high-resolution spectrum-estimation methods. Finally, a CHAD extension to spatial array-processing is still possible.

\section{APPENDIX}

This appendix provides details of the longest derivations referred to throughout the text.

\section{A. Estimation of the static channel}

With no loss of generality, the mathematical derivations will be conducted with a null Doppler compensation $(\nu=$ $0)$ and a single Doppler path $i\left(f_{\mathrm{p}_{i}} \neq 0\right)$. From (15), we then have, $\forall k \in \mathcal{I}_{K}$

$$
\begin{aligned}
\frac{1}{M} \sum_{m \in \mathcal{I}_{M}} \frac{Y_{0}^{m}[k]}{d_{k}^{m}}= & H_{0}[k]+\frac{1}{M} \sum_{m \in \mathcal{I}_{M}} \frac{\tilde{Y}_{0}^{m}[k]}{d_{k}^{m}} \\
& +\frac{1}{M} \sum_{m \in \mathcal{I}_{M}} \frac{\Xi_{0}^{m}[k]}{d_{k}^{m}} .
\end{aligned}
$$

To simplify the notations, the subscript 0 will be omitted in the sequel.

1) Study of $\frac{1}{M} \sum_{m \in \mathcal{I}_{M}} \frac{\tilde{Y}^{m}[k]}{d_{k}^{m}}$ : this sum has to be considered as a source of disturbance (in addition to the noise) for the channel $H$ estimation. Its contribution to the LOSsynchronized received signal yields

$$
\tilde{y}(t)=\alpha_{i} s\left(t-\tau_{i}\right) \mathrm{e}^{j 2 \pi f_{\mathrm{p}_{i}} t},
$$

where the relative propagation delay $\tau_{i}$ is assumed to be less than $T_{c p}$.

The received signal being sampled and cut off according to the OFDM structure, the corresponding $m^{\text {th }}$ sampled received block $\tilde{y}^{m}\left[n^{\prime}\right]$ can then be written as

$$
\begin{array}{r}
\tilde{y}^{m}\left[n^{\prime}\right]=\alpha_{i} s^{m}\left(n^{\prime} \frac{T_{u}}{K}-\tau_{i}-m T_{s}\right) \mathrm{e}^{\jmath 2 \pi f_{\mathrm{p}_{i}}\left(n^{\prime} \frac{T_{u}}{K}-\tau_{i}-m T_{s}\right),} \\
n^{\prime} \in \mathcal{I}_{K} .
\end{array}
$$

with

$s\left(n^{\prime} \frac{T_{u}}{K}-\tau_{i}-m T_{s}\right)=\frac{1}{K} \sum_{k \in \mathcal{E}_{K}} d_{k}^{m} \mathrm{e}^{\jmath 2 \pi \frac{k}{T_{u}}\left(n^{\prime} \frac{T_{u}}{K}-\tau_{i}-m T_{s}\right)}$.

It comes

$$
\begin{aligned}
\tilde{y}^{m}\left[n^{\prime}\right] & =\alpha_{i}^{\prime} \sum_{k \in \mathcal{E}_{K}} \mathrm{e}^{-\jmath 2 \pi \frac{k \tau_{i}}{T_{u}}} d_{k}^{m} \mathrm{e}^{j 2 \pi \frac{k n^{\prime}}{K}} \mathrm{e}^{\jmath 2 \pi f_{\mathrm{p}_{i}}\left(n^{\prime} \frac{T_{u}}{K}-m T_{s}\right),} \\
n^{\prime} & \in \mathcal{I}_{K},
\end{aligned}
$$

where $\alpha_{i}^{\prime}=\frac{\alpha_{i}}{K} \mathrm{e}^{-\jmath 2 \pi f_{\mathrm{p}_{i}} \tau_{i}}$.

The Doppler-shifted frequencies $f_{\mathrm{p}_{i}}$ we deal with, are assumed sufficiently low so that the phase rotation 
$\mathrm{e}^{\jmath 2 \pi f_{\mathrm{P}_{i}} n^{\prime} \frac{T_{u}}{K}}$ can be considered as constant $\left(\mathrm{e}^{32 \pi f_{\mathrm{p}_{i}} n^{\prime} \frac{T_{u}}{K}} \approx 1\right.$, $\left.\forall n \in \mathcal{I}_{K}\right)$. We can then write

$$
\begin{aligned}
\tilde{y}^{m}\left[n^{\prime}\right] & =\alpha_{i}^{\prime} \sum_{k \in \mathcal{E}_{K}} \mathrm{e}^{-\jmath 2 \pi \frac{k \tau_{i}}{T_{u}}} d_{k}^{m} \mathrm{e}^{\jmath 2 \pi \frac{k n^{\prime}}{K}} \mathrm{e}^{-\jmath 2 \pi f_{\mathrm{p}_{i}} m T_{s}}, \\
n^{\prime} & \in \mathcal{I}_{K} .
\end{aligned}
$$

Now applying a $K$-points DFT, one obtains

$$
\begin{aligned}
\tilde{Y}^{m}\left[k^{\prime}\right] & =\alpha_{i}^{\prime} \sum_{k \in \mathcal{E}_{K}} \mathrm{e}^{-\jmath 2 \pi \frac{k \tau_{i}}{T_{u}}} d_{k}^{m} \sum_{n^{\prime} \in \mathcal{I}_{K}} \mathrm{e}^{\jmath 2 \pi \frac{\left(k-k^{\prime}\right) n^{\prime}}{K}} \mathrm{e}^{-\jmath 2 \pi f_{\mathrm{p}_{i}} m T_{s}}, \\
k^{\prime} & \in \mathcal{E}_{K}
\end{aligned}
$$

Since $\sum_{n^{\prime} \in \mathcal{I}_{K}} \mathrm{e}^{\jmath 2 \pi \frac{\left(k-k^{\prime}\right) n^{\prime}}{K}}$ is null except for $k=k^{\prime}$, then $\tilde{Y}^{m}\left[k^{\prime}\right]$ simplifies to

$$
\tilde{Y}^{m}\left[k^{\prime}\right]=\alpha_{i}^{\prime \prime} d_{k^{\prime}}^{m} \mathrm{e}^{-j 2 \pi f_{\mathrm{p}_{i}} m T_{s}}, \quad k^{\prime} \in \mathcal{E}_{K},
$$

with $\alpha_{i}^{\prime \prime}=K \alpha_{i}^{\prime} \mathrm{e}^{-\jmath 2 \pi \frac{k^{\prime} \tau_{i}}{T_{u}}}$

$$
\begin{aligned}
\frac{1}{M} \sum_{m \in \mathcal{I}_{M}} \frac{\tilde{Y}^{m}\left[k^{\prime}\right]}{d_{k^{\prime}}^{m}} & =\frac{\alpha_{i}^{\prime \prime}}{M} \sum_{m \in \mathcal{I}_{M}} \mathrm{e}^{-\jmath 2 \pi f_{\mathrm{p}_{i}} m T_{s}} \\
& =\frac{\alpha_{i}^{\prime \prime}}{M} \frac{1-\exp \left(-\jmath 2 \pi f_{\mathrm{p}_{i}} M T_{s}\right)}{1-\exp \left(-\jmath 2 \pi f_{\mathrm{p}_{i}} T_{s}\right)} \\
& \propto \frac{1}{M} \frac{\sin \left(\pi f_{\mathrm{p}_{i}} M T_{s}\right)}{\sin \left(\pi f_{\mathrm{p}_{i}} T_{s}\right)}
\end{aligned}
$$

Now, since $\left|\sin \left(\pi f_{\mathrm{p}_{i}} M T_{s}\right)\right| \leq 1$, it comes

$$
\lim _{M \rightarrow+\infty} \frac{1}{M} \sum_{m \in \mathcal{I}_{M}} \frac{\tilde{Y}^{m}[k]}{d_{k}^{m}}=0 .
$$

2) Study of $\frac{1}{M} \sum_{m \in \mathcal{I}_{M}} \frac{\Xi^{m}[k]}{d_{k}^{m}}$ : from the strong law of large numbers, one has (see [18] pp. 151-152)

$$
\frac{1}{M} \sum_{m \in \mathcal{I}_{M}} \frac{\Xi^{m}[k]}{d_{k}^{m}} \underset{M \rightarrow+\infty}{\stackrel{a . s .}{\longrightarrow}} \mathrm{E}\left\{\frac{\Xi^{m}[k]}{d_{k}^{m}}\right\} .
$$

The zero-mean random variables $\Xi^{m}[k]$ and $\frac{1}{d_{k}^{m}}$ being uncorrelated, it comes

$$
\mathrm{E}\left\{\frac{\Xi^{m}[k]}{d_{k}^{m}}\right\}=\mathrm{E}\left\{\Xi^{m}[k]\right\} \mathrm{E}\left\{\frac{1}{d_{k}^{m}}\right\}=0
$$

and consequently

$$
\lim _{M \rightarrow+\infty} \frac{1}{M} \sum_{m \in \mathcal{I}_{M}} \frac{\Xi^{m}[k]}{d_{k}^{m}} \stackrel{\text { a.s. }}{=} 0 .
$$

B. Study of the cross-correlation between $\mathcal{X}^{e}$ and $\mathcal{X}^{d}$

One has using (28) both with $k=k^{\prime}$ and $k \neq k^{\prime}$ :

$$
\begin{aligned}
& \mathrm{E}\left\{\mathcal{X}^{\mathrm{e}}\left(\mathcal{X}^{\mathrm{d}}\right)^{*}\right\}= \\
& \mathrm{E}\left\{\frac{s_{k_{1} k_{1}}\left(f_{\mathrm{p}_{i}}\right)}{M K \# \# \mathcal{E}_{K}} \sum_{m_{1} \in \mathcal{I}_{M}} \mathrm{e}^{\jmath 2 \pi\left(f_{\mathrm{p}_{i}}-\nu\right) m_{1} T_{s}}\right. \\
& \sum_{k_{1} \in \mathcal{E}_{K}}\left|d_{k_{1}}^{m_{1}}\right|^{2} \mathrm{e}^{\frac{j \pi\left(n-n_{i}\right) k_{1}}{K}} \\
& \frac{1}{M K \# \mathcal{E}_{K}} \sum_{m_{2} \in \mathcal{I}_{M}} \mathrm{e}^{-\jmath 2 \pi\left(f_{\mathrm{p}_{i}}-\nu\right) m_{2} T_{s}} \\
& \left.\sum_{k_{2}, k_{2}^{\prime} \neq k_{2}}\left(d_{k_{2}}^{m_{2}}\right)^{*} d_{k_{2}^{\prime}}^{m_{2}} \mathrm{e}^{\frac{-\jmath 2 \pi\left(n k_{2}^{\prime}-n_{i} k_{2}\right)}{K}} s_{k_{2} k_{2}^{\prime}}\left(f_{\mathrm{p}_{i}}\right)\right\} .
\end{aligned}
$$

Since $E\left\{\left|d_{k_{1}}^{m_{1}}\right|^{2}\left(d_{k_{2}}^{m_{2}}\right)^{*} d_{k_{2}^{\prime}}^{m_{2}}\right\}=0$ for any triplet $\left(k_{1}, k_{2}, k_{2}^{\prime}\right)$ such that $k_{2} \neq k_{2}^{\prime}$, we get

$$
\mathrm{E}\left\{\mathcal{X}^{\mathrm{e}}\left(\mathcal{X}^{\mathrm{d}}\right)^{*}\right\}=0
$$

and the random variables $\mathcal{X}^{\mathrm{e}}$ and $\mathcal{X}^{\mathrm{d}}$ are then uncorrelated.

\section{CAF peak shape - equation (34)}

Since $s_{k k}\left(f_{\mathrm{p}_{i}}\right)$ does not depend on $k$, it comes using (28) with $k=k^{\prime}$ :

$$
\begin{aligned}
& \mathrm{E}\left\{\mathcal{X}^{\mathrm{e}}[n, \nu]\right\}=\frac{\gamma s_{k k}\left(f_{\mathrm{p}_{i}}\right)}{M K \# \mathcal{E}_{K}} \sum_{m \in \mathcal{I}_{M}} \mathrm{e}^{\jmath 2 \pi\left(f_{\mathrm{p}_{i}}-\nu\right) m T_{s}} \sum_{k=K_{\mathrm{m}}}^{K_{\mathrm{M}}} \mathrm{e}^{\frac{j 2 \pi\left(n-n_{i}\right) k}{K}} \\
& =\frac{\gamma s_{k k}\left(f_{\mathrm{p}_{i}}\right)}{M K \# \mathcal{E}_{K}} \sum_{m \in \mathcal{I}_{M}} \mathrm{e}^{\jmath 2 \pi\left(f_{\mathrm{p}_{i}}-\nu\right) m T_{s}} \mathrm{e}^{\frac{j 2 \pi\left(n-n_{i}\right) K_{\mathrm{m}}}{K}} \sum_{k=0}^{K_{\mathrm{M}}-K_{\mathrm{m}}} \mathrm{e}^{\frac{\jmath 2 \pi\left(n-n_{i}\right) k}{K}} \\
& =\frac{\gamma s_{k k}\left(f_{\mathrm{p}_{i}}\right)}{M K \# \mathcal{E}_{K}} \mathrm{e}^{\jmath \pi\left(f_{\mathrm{p}_{i}}-\nu\right)(M-1) T_{s}} \mathrm{e}^{\jmath \pi\left(n-n_{i}\right) \frac{K_{\mathrm{M}}+K_{\mathrm{m}}-1}{K}} \\
& \frac{\sin \pi\left(f_{\mathrm{p}_{i}}-\nu\right) M T_{s}}{\sin \pi\left(f_{\mathrm{p}_{i}}-\nu\right) T_{s}} \frac{\sin \pi\left(n-n_{i}\right) \# \mathcal{E}_{K} / K}{\sin \pi\left(n-n_{i}\right) / K}
\end{aligned}
$$

Taking the squared modulus, then using (29) and $|\gamma|=1$, we finally obtain the expression of the detection peak mean shape

$$
\begin{aligned}
& \left|\mathrm{E}\left\{\mathcal{X}^{\mathrm{e}}[n, \nu]\right\}\right|^{2}= \\
& \frac{\frac{\sin ^{2} \pi f_{\mathrm{p}_{i}} T_{u}}{\sin ^{2} \pi f_{\mathrm{p}_{i}} T_{u} / K}}{\left(M K \# \mathcal{E}_{K}\right)^{2}} \frac{\sin ^{2} \pi\left(f_{\mathrm{p}_{i}}-\nu\right) M T_{s}}{\sin ^{2} \pi\left(f_{\mathrm{p}_{i}}-\nu\right) T_{s}} \frac{\sin ^{2} \pi\left(n-n_{i}\right) \# \mathcal{E}_{K} / K}{\sin ^{2} \pi\left(n-n_{i}\right) / K} .
\end{aligned}
$$


D. CAF clutter floor mean level - equation (36)

One has, using (28) with $k=k^{\prime}$ :

$$
\begin{aligned}
& \operatorname{Var}\left\{\mathcal{X}^{\mathrm{e}}\right\}=\mathrm{E}\left\{\left|\mathcal{X}^{\mathrm{e}}-\mathrm{E}\left\{\mathcal{X}^{\mathrm{e}}\right\}\right|^{2}\right\} \\
& =\mathrm{E}\left\{\mid \frac{s_{k k}\left(f_{\mathrm{p}_{i}}\right)}{M K \# \mathcal{E}_{K}} \sum_{m \in \mathcal{I}_{M}} \mathrm{e}^{\jmath 2 \pi\left(f_{\mathrm{p}_{i}}-\nu\right) m T_{s}}\right. \\
& \left.\left.\sum_{k \in \mathcal{E}_{K}}\left(\left|d_{k}^{m}\right|^{2}-\mathrm{E}\left\{\left|d_{k}^{m}\right|^{2}\right\}\right) \mathrm{e}^{\frac{j 2 \pi\left(n-n_{i}\right) k}{K}}\right|^{2}\right\} \\
& =\frac{\left|s_{k k}\left(f_{\mathrm{p}_{i}}\right)\right|^{2}}{\left(M K \# \mathcal{E}_{K}\right)^{2}} \sum_{m, m^{\prime} \in \mathcal{I}_{M}^{2}} \mathrm{e}^{\jmath 2 \pi\left(f_{\mathrm{p}_{i}}-\nu\right)\left(m-m^{\prime}\right) T_{s}} \\
& \sum_{k, k^{\prime} \in \mathcal{E}_{K}^{2}} \mathrm{E}\left\{\left(\left|d_{k}^{m}\right|^{2}-\mathrm{E}\left\{\left|d_{k}^{m}\right|^{2}\right\}\right)\left(\left|d_{k^{\prime}}^{m^{\prime}}\right|^{2}-\mathrm{E}\left\{\left|d_{k^{\prime}}^{m^{\prime}}\right|^{2}\right\}\right)\right\} \\
& \mathrm{e}^{\jmath 2 \pi\left(n-n_{i}\right) \frac{\left(k-k^{\prime}\right)}{K}}
\end{aligned}
$$

This expression is clearly null for $m \neq m^{\prime}$ or $k \neq k^{\prime}$ and one finally obtains

$$
\begin{aligned}
& \operatorname{Var}\left\{\mathcal{X}^{\mathrm{e}}[n, \nu]\right\} \\
& =\frac{\mid s_{k k}\left(\left.f_{\mathrm{p}_{i}}\right|^{2}\right.}{\left(M K \# \mathcal{E}_{K}\right)^{2}} \sum_{m \in \mathcal{I}_{M}} \sum_{k \in \mathcal{E}_{K}}\left(\mathrm{E}\left\{\left|d_{k}^{m}\right|^{4}\right\}-\mathrm{E}\left\{\left|d_{k}^{m}\right|^{2}\right\}^{2}\right) \\
& =\frac{\frac{\sin ^{2} \pi f_{\mathrm{p}_{i}} T_{u}}{\sin ^{2} \pi f_{\mathrm{p}_{i}} T_{u} / K}}{M K^{2} \# \mathcal{E}_{K}}\left(\mathrm{E}\left\{\left|d_{k}^{m}\right|^{4}\right\}-1\right) .
\end{aligned}
$$

\section{E. CAF clutter floor level - equation (37)}

Using (28) with $k \neq k^{\prime}$, one has:

$$
\begin{aligned}
& \operatorname{Var}\left\{\mathcal{X}^{\mathrm{d}}\right\}= \\
& \frac{1}{\left(M K \# \mathcal{E}_{K}\right)^{2}} \sum_{m \in \mathcal{I}_{M}} \mathrm{e}^{j 2 \pi\left(f_{\mathrm{p}_{i}}-\nu\right) m T_{s}} \\
& \mathrm{E}\left\{\sum_{k_{1}, k_{1}^{\prime} \neq k_{1} \in \mathcal{E}_{K}^{2}} d_{k_{1}}^{m}\left(d_{k_{1}^{\prime}}^{m}\right)^{*} \mathrm{e}^{\frac{j 2 \pi\left(n k_{1}^{\prime}-n_{i} k_{1}\right)}{K}} s_{k_{1} k_{1}^{\prime}}\left(f_{\mathrm{p}_{i}}\right)\right. \\
& \sum_{m^{\prime} \in \mathcal{I}_{M}} \mathrm{e}^{-\jmath 2 \pi\left(f_{\mathrm{p}_{i}}-\nu\right) m^{\prime} T_{s}} \\
& \left.\sum_{k_{2}, k_{2}^{\prime} \neq k_{2} \in \mathcal{E}_{K}^{2}} d_{k_{2}^{\prime}}^{m^{\prime}}\left(d_{k_{2}}^{m^{\prime}}\right)^{*} \mathrm{e}^{\frac{-\jmath 2 \pi\left(n k_{2}^{\prime}-n_{i} k_{2}\right)}{K}}\left(s_{k_{2} k_{2}^{\prime}}\left(f_{\mathrm{p}_{i}}\right)\right)^{*}\right\},
\end{aligned}
$$

since $\mathrm{E}\left\{d_{k_{1}}^{m}\left(d_{k_{1}^{\prime}}^{m}\right)^{*}\left(d_{k_{2}}^{m^{\prime}}\right)^{*} d_{k_{2}^{\prime}}^{m{ }^{\prime}}\right\}=0$ for $m \neq m^{\prime}$ and since a QAM signal is second-order circular $\left(\mathrm{E}\left\{\left(d_{k}^{m}\right)^{2}\right\}=0\right)$ [19], only the terms with $k_{1}=k_{2}$ and $k_{1}^{\prime}=k_{2}^{\prime}$ remain. Hence, it comes using $\left(\operatorname{Var}\left\{d_{k}^{m}\right\}\right)^{2}=1$ :

$$
\operatorname{Var}\left\{\mathcal{X}^{\mathrm{d}}\right\}=\frac{1}{M K^{2}\left(\# \mathcal{E}_{K}\right)^{2}} \sum_{k \in \mathcal{E}_{K}} \sum_{k^{\prime} \in \mathcal{E}_{K}, k^{\prime} \neq k} s_{k k^{\prime}}^{2}\left(f_{\mathrm{p}_{i}}\right) .
$$

\section{F. Equation (41)}

$$
\begin{aligned}
H^{m}\left[k^{\prime}\right] \triangleq & \frac{Y^{m}\left[k^{\prime}\right]}{\sqrt{\# \mathcal{E}_{K}} d_{k^{\prime}}^{m}} \\
= & \sum_{n^{\prime}, k \in \mathcal{I}_{K} \times \mathcal{E}_{K}} \frac{d_{k}^{m}}{\# \mathcal{E}_{K} d_{k^{\prime}}^{m}} \mathrm{e}^{\frac{\jmath 2 \pi\left(n^{\prime}-n_{i}\right) k}{K}} \\
= & \mathrm{e}^{\jmath 2 \pi f_{\mathrm{p}_{i}}\left(\frac{\left(n^{\prime}-n_{i}\right) T_{u}}{K}+m T_{s}\right)} \mathrm{e}^{\frac{-\jmath 2 \pi n^{\prime} k^{\prime}}{K}} \\
= & \mathrm{e}^{\jmath 2 \pi f_{\mathrm{p}_{i}}\left(\frac{-n_{i} T_{u}}{K}+m T_{s}\right)} \sum_{k \in \mathcal{E}_{K}} \frac{d_{k}^{m}}{\# \mathcal{E}_{K} d_{k^{\prime}}^{m}} \mathrm{e}^{\frac{-\jmath 2 \pi n_{i} k}{K}} \\
& \sum_{n^{\prime} \in \mathcal{I}_{K}} \mathrm{e}^{\jmath 2 \pi\left(\frac{k-k^{\prime}}{K}+f_{\mathrm{p}_{i}} \frac{T_{u}}{K}\right) n^{\prime}} .
\end{aligned}
$$

We recognize in the latter summation, the expression of the function $s_{k k^{\prime}}\left(f_{\mathrm{p}_{i}}\right)$ defined in (29). It comes

$$
H^{m}\left[k^{\prime}\right]=\frac{\gamma}{\# \mathcal{E}_{K}} \mathrm{e}^{\jmath 2 \pi f_{\mathrm{p}_{i}}\left(m T_{s}\right)} \sum_{k \in \mathcal{E}_{K}} \frac{d_{k}^{m}}{d_{k^{\prime}}^{m}} \mathrm{e}^{\frac{-j 2 \pi n_{i} k}{K}} s_{k k^{\prime}}\left(f_{\mathrm{p}_{i}}\right),
$$

where $\gamma=\mathrm{e}^{\frac{-\jmath 2 \pi f_{\mathrm{p}_{i}} n_{i} T_{u}}{K}}$.

\section{REFERENCES}

[1] P. Forster and M. Lesturgie, "Passive radar for target tracking and classification," Proc. of the IEEE-RADAR 2004, p. inconnues, 2004.

[2] H. Griffiths and C. Baker, "Passive coherent location radar systems. part 1: performance prediction," in Radar, Sonar and Navigation, IEE Proceedings-, vol. 152, pp. 153-159, IET, 2005.

[3] K. Kulpa, J. Misiurewicz, M. Malanowski, P. Samczyński, and M. Smolarczyk, "Recent developments in passive radars," Proceedings of Military Sensors, 2009.

[4] "ETSI Standard : EN 300744 V1.5.1 Digital Video Broadcasting (DVB),"

[5] G. Gassier, F. Briolle, J. Barrère, and G. Chabriel, "Pilot-Aided Beamforming for OFDM Detection Over Severe Multipath Fading," in 21st IEEE International Conference on Electronics Circuits and Systems, (Marseille), pp. 247-250, December 2014.

[6] F. Colone, "A Multistage Processing Algorithm for Disturbance Removal and Target Detection in Passive Bistatic Radar," IEEE Transaction on Aerospace and Electronic Systems, vol. 45, no. 2, April 2008.

[7] M. Meller, "Cheap Cancellation of Strong Echoes for Digital Passive and Noise Radars," IEEE Transactions on Signal Processing, vol. 60, no. 5, April 2012.

[8] D. Poullin, "Passive Radar using COFDM (DAB or DVB-T) Broadcasters as Opportunistic Illuminators". InTech, ISBN 978953-7619-70-1, February 2010.

[9] G. Chabriel, J. Barrère, G. Gassier, and F. Briolle, "Passive Covert Radars using CP-OFDM signals A new efficient method to extract targets echos," in RADAR 2014, IEEE International Conference, (Lille), pp. 1-6, October 2014.

[10] J. Palmer, H. Harms, S. Searle, and L. Davis, "DVB-T Passive Radar Signal Processing," IEEE Transactions on Signal Processing, vol. 61, no. 8, pp. 2116 - 2126, April 2013.

[11] H. Harms, L. Davis, and J. Palmer, "Understanding the signal structure in DVB-T signals for passive radar detection," in IEEE Radar Conference, (Washington, DC), pp. 532 - 537, May 2010.

[12] M. Glende, "PCL signal processing for sidelobe reduction in case of periodical illuminator signals," in International Radar Symposium, (Krakow), pp. 1-4, 24-26 May 2006.

[13] S. Searle, J. Palmer, L. Davis, D. O'Hagan, and M. Ummenhofer, "Evaluation of the ambiguity function for passive radar with ofdm transmissions," in 2014 IEEE Radar Conference, (Cincinnati, OH), pp. 1040-1045, 19-23 May 2014. 
[14] C. Berger, B. Demissie, J. Heckenbach, P. Willett, and Z. Shengli, "Signal Processing for Passive Radar Using OFDM Waveforms," IEEE Journal of Selected Topics in Signal Processing, vol. 4, no. 1, pp. 226-238, Feb. 2010.

[15] C. Moscardini, D. Petri, A. Capria, M. Conti, M.and Martorella, and F. Berizzi, "Batches algorithm for passive radar: a theoretical analysis," IEEE Transactions on Aerospace and Electronic Systems, vol. 51, pp. 1475-1487, April 2015.

[16] X. P. E. Howland, H. D. Griffiths, and C. J. Baker, Bistatic Radar: Emerging Technology. John Wiley \& Sons, 2008.

[17] S. Wei, D. L. Goeckel, and P. A. Kelly, "Convergence of the Complex Envelope of Bandlimited OFDM Signals," IEEE Transactions on Information Theory, vol. 56, no. 10, pp. 4893 - 4904, April 2010.

[18] A. Karr, Probability. Springer-Verlag, 1993.

[19] T. Adali, P. Schreier, and L. Scharf, "Complex-Valued Signal Processing: The Proper Way to Deal With Impropriety," IEEE Transactions on Signal Processing, vol. 59, no. 11, pp. 5101 5125, November 2011.

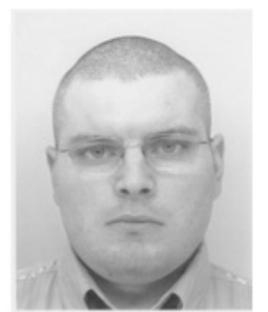

Ghislain Gassier received his Master degree in applied mathematics (Statistics - Data Processing) from the Aix Marseille Université (AMU), France, in 2010, and his Ph.D. degree from the Université de Toulon (UTLN), France, in 2016. He has been working as a teaching assistant for the French Ministry of Defence (French Air Force Academy Ecole de l'Air) and did his research activities both in the Morpho-Analysis in Signal Team (MAS'Air) of the Research Center of the French Air Force (CReA), Salon-De-Provence, and in the Signals and Systems Team of IM2NP Laboratory, Toulon, France.



Gilles Chabriel received the Microelectronic and Automatic Engineer diploma from the Institut des Sciences de l'Ingénieur de Montpellier (formerly ISIM, currently Polytech'Montpellier), Montpellier, France, in 1992 and the Ph.D. degree in engineering sciences from the Université de Toulon (UTLN), La Garde, France, in 1997.

$\mathrm{He}$ is currently an Assistant Professor in the Sciences and Applied Sciences Department of UTLN, where he teaches Applied Mathematics, Electronics and Signal Processing. In the Signals and Systems Team of the Institut Matériaux, Microélectronique, Nanosciences de Provence (IM2NP, UMR CNRS 7334), his research is in the areas of signal processing, telecommunication, passive radar and electronic systems.

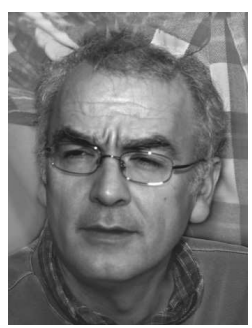

Jean Barrère was born in Bayonne, France, in 1961. He received the Ph.D. degree in mechanics from the University of Bordeaux, France, in January 1990. From 1990 to 1994, he worked for ELF-Aquitaine (currently TotalFina-Elf), CERFACS (European Centre for Research and Advanced Training in Scientific Computation), and Aerospatiale (currently Airbus), all in Toulouse, France. His work focused on filtration laws in porous media studies and then plane structure design. He is currently a research engineer in the Signals and Systems Team of IM2NP Laboratory, Université de Toulon (UTLN), La Garde, France, where his research interests include wave separation, array processing and passive radar.



Françoise Briolle received the Habilitation à Diriger des Recherches (HDR) from the Aix Marseille Université (AMU), Marseille, France. She works as an associate professor in the Sciences Department of AMU where she teaches signal processing and telecommunications. In the Dynamical System team of the Theoretical Physics Center (CPT), her research was mainly focused on non-linear systems. Since 2010 she is seconded in the Research Center of the French Air Force (CReA) where her research is dedicated to signal processing and passive radar.
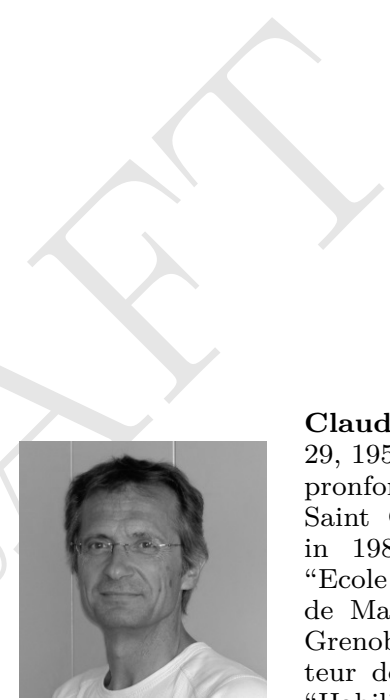

Claude Jauffret born in France on March 29, 1957, received the "Diplôme d'Etudes Appronfondies" in Applied Mathematics, from Saint Charles University, Marseille, France, in 1981; the "Diplôme d'Ingénieur" from "Ecole Nationale Supérieure d'Informatique et de Mathématiques Appliqués de Grenoble", Grenoble, France, in 1983Âă; the title of "Docteur de l'Université" Âă in 1993Âă; and the "Habilitation à Diriger des Recherches" from the University of Toulon and Var, France. From Nov. 1983 to Nov. 1988, he worked on passive sonar systems; more precisely, on target motion analysis at GERDSM, France. After a sabbatical year at the University of Connecticut (from Nov. 1988 to Dec. 1989), during which he worked on tracking problems in a cluttered environment, he developed research in tracking, data fusion, and extraction in GERDSM. Since Sept. 1996, he has been at the University of Toulon, where he teaches statistical signal processing. His current research is on observability estimation in nonlinear systems, as it appears in tracking problems. 